\title{
TITLE:
}

\section{Characterization of near-wall turbulence in terms of equilibrium and "'bursting"' solutions}

\section{AUTHOR(S):}

Jiménez, Javier; Kawahara, Genta; Simens, Mark P.; Nagata, Masato; Shiba, Makoto

\section{CITATION:}

Jiménez, Javier ...[et al]. Characterization of near-wall turbulence in terms of equilibrium and "'bursting" solutions. PHYSICS OF FLUIDS 2005, 17(1): 015105

\section{ISSUE DATE:}

2005-01

URL:

http://hdl.handle.net/2433/39786

\section{RIGHT:}

Copyright 2005 American Institute of Physics. This article may be downloaded for personal use only. Any other use requires prior permission of the author and the American Institute of Physics. 


\title{
Characterization of near-wall turbulence in terms of equilibrium and "bursting" solutions
}

\author{
Javier Jiménez ${ }^{\mathrm{a})}$ \\ School of Aeronautics, Universidad Politécnica, 28040 Madrid, Spain and Center for Turbulence Research, \\ Stanford University, California 94305
}

Genta Kawahara

Department of Aeronautics and Astronautics, Advanced Research Institute of Fluid Science and Engineering, Kyoto University, Kyoto 606-8501, Japan

Mark P. Simens

School of Aeronautics, Universidad Politécnica, 28040 Madrid, Spain

Masato Nagata

Department of Aeronautics and Astronautics, Advanced Research Institute of Fluid Science and Engineering, Kyoto University, Kyoto 606-8501, Japan

Makoto Shiba

Department of Mechanical Engineering, Ehime University, Matsuyama 790-8577, Japan

(Received 12 April 2004; accepted 13 September 2004; published online 15 December 2004)

\begin{abstract}
Near-wall turbulence in the buffer region of Couette and Poiseuille flows is characterized in terms of recently-found nonlinear three-dimensional solutions to the incompressible Navier-Stokes equations for wall-bounded shear flows. The data suggest that those solutions can be classified into two families, of which one is dominated by streamwise vortices, and the other one by streaks. They can be associated with the upper and lower branches of the equilibrium solutions for Couette flow found by Nagata ["Three-dimensional finite-amplitude solutions in plane Couette flow: Bifurcation from infinity," J. Fluid Mech. 217, 519 (1990)]. The quiescent structures of near-wall turbulence are shown to correspond to the vortex-dominated family, but evidence is presented that they burst intermittently both in minimal and in fully turbulent flows. The intensity and period of the bursts are Reynolds-number dependent, but they saturate at high enough Reynolds numbers. The time-periodic exact solution found for Couette flow by Kawahara and Kida ["Periodic motion embedded in plane Couette turbulence: Regeneration cycle and burst," J. Fluid Mech. 449, 291 (2001)] can be used as a simplified model for the bursting process. (C) 2005 American Institute of Physics.
\end{abstract}

[DOI: $10.1063 / 1.1825451]$

\section{INTRODUCTION}

Wall-bounded flows have been important in turbulence research ever since the famous 1883 experiments by Reynolds. This is particularly true of the immediate near-wall layer which, because its Reynolds number is locally low, is usually considered to be a good candidate for an approximate description in terms of simple deterministic structures.

Nonlinear equilibrium solutions of the three-dimensional Navier-Stokes equations, with characteristics which suggest that they may be useful in such a description, have been obtained numerically in the past few years for plane Couette flow, ${ }^{1,2}$ plane Poiseuille flow, ${ }^{2-4}$ and an autonomous wall flow. ${ }^{5}$ All those solutions look qualitatively similar, ${ }^{6,7}$ and take the form of a wavy low-velocity streak flanked by staggered quasistreamwise vortices of alternating signs, resembling the spatially-coherent objects educed from the nearwall region of true turbulent flows. ${ }^{8,9}$ The mean and fluctuation intensity profiles of the equilibrium structures are also reminiscent of the experimental values. ${ }^{2,5}$ In those cases

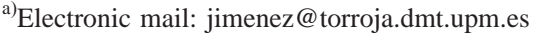

in which their stability has been investigated, the equilibrium solutions are unstable saddles in phase space at the Reynolds numbers at which turbulence is observed. They are not therefore expected to be found as such in real turbulence but, since the velocity of the system in phase space vanishes at fixed points, whether stable or not, any turbulent flow could spend a substantial fraction of its lifetime in their neighborhood.

Although the observed similarities suggest that all those structures are related to each other and to self-sustaining wall turbulence, the nature of those relations is unclear. The first goal of this paper is to clarify that point by comparing as many as possible of the known equilibrium solutions, among themselves and with real near-wall turbulence. This comparison will also include the time-periodic saddle orbits identified by Kawahara and Kida, ${ }^{10}$ which not only approximate the profiles of average velocity and of the intensities of nearwall turbulence, but also part of its temporal structure.

In fact, the second problem that we will consider is the possible relation between temporal intermittency in the nearwall layer and such time-dependent simple solutions of the Navier-Stokes equations. The term "burst" was originally 
introduced to describe fluid eruptions observed near the wall in the early visualizations of turbulent boundary layers. ${ }^{11}$ It was initially hypothesized that bursts were due to the intermittent breakup of the near-wall streaks, but even the original authors later acknowledged that their visualizations could be consistent with permanent advecting objects, ${ }^{12}$ and the term became eventually associated with the ejections observed by stationary velocity probes. With the advent of numerical simulations, it became apparent that the streaks were long-lived streamwise velocity structures, and that the sweeps and ejections identified in the analysis of single-point data were mostly due to the passing of shorter quasistreamwise vortices, intermittent in space but not necessarily in time. ${ }^{13}$ The question of whether the observed temporally intermittent sublayer events were visualization artifacts or really existed in the near-wall layer was bypassed by this explanation.

The well-documented mutual dependence of the nearwall streaks and vortices is consistent both with equilibrium models sustained by steady nonlinear interactions, such as in the structures mentioned above, and with temporal cycles in which both types of structures periodically create each other. The difficulty of following for long times individual structures in fully turbulent flows complicates the experimental or numerical distinction between essentially permanent objects and intrinsically time-dependent processes with a long period, but intermittent breakdown of near-wall turbulence is observed in minimal-flow numerical simulations for which spatial intermittency is not an issue. ${ }^{5,14}$ The same is true of autonomous wall flows in which the observation is simplified by the small wall-normal dimensions of the simulation domain. ${ }^{15}$

By comparing periodic solutions such those in Ref. 10, minimal simulations, and fully turbulent ones, we will try to clarify whether intermittent behavior, as distinguished from the vortex-passing bursts, is found in fully turbulent flows, and whether it can be explained in terms of simple timeperiodic solutions.

We will also address the question of whether the characteristics of such equilibrium or periodic solutions can explain the wavelength-selection properties of near-wall turbulence, such as the well-known mean streak separation of $z^{+} \approx 100$ or the equally intriguing $x^{+} \approx 300$ streamwise separation found in turbulent flows between vortex pairs within the same streak. ${ }^{14}$

To simplify the discussion, stationary or traveling permanent waves, and solutions which can be reduced to limit cycles in some frame of reference, will be referred to as "simple" from now on. Of those, the permanent waves and the turbulent flows whose statistics are roughly similar to them, will be denoted as "quiescent." Solutions with stronger vorticity, usually corresponding to a fast evolution in phase space, will be called "excited."

Some of the older solutions required recomputing for the purpose of this paper, using numerical methods which are occasionally slightly different from the original ones. Those methods are described in Sec. II. The comparison between the different equilibrium and periodic solutions is made in Sec. III, and their relation with fully developed turbulence is discussed in Sec. IV. Temporal intermittency is discussed in Sec. V, and conclusions are offered in Sec. VI. A preliminary version of part of the present paper appeared previously as Ref. 16.

\section{COMPUTATIONAL METHODS}

\section{A. Autonomous solutions}

The permanent traveling-wave solutions described below as "autonomous" are computed using a slightly modified version ${ }^{17}$ of the numerical scheme used in Refs. 5 and 15. The flow is established in a numerical domain with spatial periodicities $L_{x}$ and $L_{z}$ in the streamwise and in the spanwise directions, over a wall located at $y=0$. The streamwise, wallnormal, and spanwise velocity components are $u, v$, and $w$. The Navier-Stokes equations are integrated in the form of evolution equations for the wall-normal vorticity $\omega_{y}$ and for $\nabla^{2} v$, using a pseudospectral code with Fourier expansions in the two wall-parallel directions and Chebychev polynomials in the wall-normal direction. ${ }^{18}$ At each time step the righthand sides of the two evolution equations are multiplied by a damping mask $1-\Delta t F(y)$, where

$$
F(y)=0 \text { if } y \leqslant \delta_{1}, \quad F(y)=1 / \theta \text { if } y \geqslant \delta_{2}=1.5 \delta_{1},
$$

and the two limits of $F(y)$ are connected smoothly by a cubic spline. This mask can be interpreted as a linear dissipation for each of the two evolution variables. The decay time $\theta$ is chosen so that all the vorticity fluctuations are effectively damped above $y \approx\left(\delta_{1}+\delta_{2}\right) / 2$. The equations are not modified below the mask lower limit $\delta_{1}$. Irrotational fluctuations are not affected anywhere, and the outer edge of the NavierStokes layer is bounded by a potential core which prevents the formation of viscous boundary layers at the mask boundary. No-slip, impermeable boundary conditions are imposed at the wall.

While the flows in Refs. 5 and 15 were integrated at constant mass flux in a channel, the present computations were initially carried out at constant driving stress in a "semi-infinite" domain. The velocities were matched to outer potential fluctuations extending to infinity from the edge, $y$ $=h>\delta_{2}$, of the computational domain. ${ }^{19}$ This driving mechanism is free from the complications of a "second wall" across the potential layer, and, in particular, from the effect of a mean pressure gradient, and should, in principle, be preferable to simulations involving two-walled channels. The total shear stress, for example, is constant across the NavierStokes layer instead of varying linearly across the channel, and the only Reynolds number in the problem is $\delta_{1}^{+}$. The superscript + denotes "wall" variables normalized with the kinematic viscosity $\nu$ and with the friction velocity $u_{\tau}$.

This driving mechanism had been successfully used to simulate autonomous wall flows in large computational boxes ${ }^{17}$ but, in the present case, it failed to reproduce the simple solutions found by Jiménez and Simens ${ }^{5}$ in a pressure-driven channel. The flow passed directly from fully chaotic (minimal) turbulence to laminar decay upon minor changes in the parameters.

It was therefore decided to reintroduce some pressure effects. The basic structure of the code is maintained, and, in 
TABLE I. Parameters of the autonomous simulations used in the text. $L_{x}$ and $L_{z}$ are the box dimensions, $U_{c}$ is the phase velocity, and $u_{\max }^{\prime}, v_{\max }^{\prime}$, and $\omega_{x, \max }^{\prime}$ are the maximum fluctuation intensities used below to characterize solutions.

\begin{tabular}{cccccccc}
\hline \hline & $L_{x}^{+}$ & $L_{z}^{+}$ & $\delta_{1}^{+}$ & $U_{c}^{+}$ & $u_{\max }^{\prime+}$ & $v_{\max }^{\prime+}$ & $\omega_{x, \max }^{\prime+}$ \\
\hline $\mathrm{A} 1$ & 189 & 180 & 42.0 & 12.6 & 2.71 & 0.616 & 0.116 \\
$\mathrm{~A} 2$ & 189 & 180 & 45.6 & 12.4 & 2.84 & 0.612 & 0.117 \\
$\mathrm{~A} 3$ & 168 & 180 & 38.4 & 13.2 & 2.54 & 0.592 & 0.124 \\
$\mathrm{~A} 4$ & 168 & 180 & 42.0 & 12.8 & 2.59 & 0.598 & 0.121 \\
$\mathrm{~A} 5$ & 151 & 180 & 42.0 & 13.2 & 2.51 & 0.578 & 0.123 \\
\hline \hline
\end{tabular}

particular, the driving mechanism for the mean flow is still a constant shear far from the wall, instead of a fixed imposed mean pressure gradient. The mean velocity profile is linear far from the wall, rather than parabolic, but the potential fluctuations in the masked region are required to match a no-stress impermeable boundary at $y=H>h$ instead of decaying at $y \rightarrow \infty$. All the cases presented in this paper were computed with $H=2 h$ and with the viscosity adjusted so that $h^{+}=120$.

This modification introduces a small fluctuating pressure gradient which maintains the instantaneous mass flow constant across the domain $(0, H)$. It was found to be sufficient to restore the existence of steady traveling waves. Their computational parameters are summarized in Table I. They were computed using $48 \times 49 \times 48$ spectral modes, before dealiasing. The resulting resolution is $\Delta x^{+} \approx \Delta z^{+} \approx 4$, with a maximum grid spacing $\Delta y^{+} \approx 3$ below the mask.

The significance of this observation is not clear, although it is not surprising that the properties of constant-mass and constant-stress simulations should differ in small computational domains. Note that the solutions discussed in this section differ from others used in this paper in that they are obtained from an initial-value problem, and are therefore stable with a nonzero basin of attraction. This explains their sensitivity to the stress boundary condition, which changes their stability although not their qualitative character. The present traveling waves do not differ visually from those in Ref. 5, even though the codes are different. The chaotic solutions obtained from the constant-stress boundary conditions are also indistinguishable from the chaotic solutions obtained by raising the mask either in the present code or in Ref. 5. They all agree with minimal Poiseuille flows below roughly half the mask height. ${ }^{5,17}$ Because of the presence of a required fluctuation of the spatially-constant pressure gradient, these flows are classified below as part of the Poiseuille family, even if the temporal average of their mean pressure gradient is zero.

\section{B. Plane Couette solutions}

Nagata's steady solutions ${ }^{1}$ for incompressible plane Couette flow were recomputed using a method similar to that in Ref. 20. The flow is described by its deviation $\left(u^{*}, v, w\right)$ from the linear profile, and is obtained numerically by solving steady nonlinear equations for the streamwise velocity $u_{0}(y)=\left\langle u^{*}\right\rangle$, where \langle\rangle denotes averaging over a wall-parallel plane, and for $\omega_{y}$ and $\nabla^{2} v$, as in the preceding section. The solutions are expressed as double Fourier expansions in the two wall-parallel directions. The wall-normal expansions are expressed in terms of the centered dimensionless coordinate $y^{*}=y / h-1$, where $y^{*}=0$ is the midplane of the channel and $h$ is half the wall separation. They use the basis functions

$$
\left(1-y^{* 2}\right) T_{l}\left(y^{*}\right)
$$

for $u_{0}$ and $\omega_{y}$, and

$$
\left(1-y^{* 2}\right)^{2} T_{l}(y *)
$$

for $v$, where $T_{l}\left(y^{*}\right)$ is the $l$ th order Chebychev polynomial. They satisfy the boundary conditions

$$
u_{0}=\omega_{y}=v=\frac{\partial v}{\partial y}=0 \text { at } y^{*}= \pm 1 .
$$

The collocation method with grid points $y^{*}=\cos [m \pi /(M$ $+1)],(m=1,2, \ldots, M)$ is used to construct a system of quadratic equations for the Fourier-Chebychev-Fourier coefficients, which is solved by the Newton-Raphson method. The arc-length method ${ }^{20}$ is used to track the nonlinear solutions, with the three parameters $\operatorname{Re}, L_{x}$, and $L_{z}$ being changed independently.

It is well-known that a laminar plane Couette flow is linearly stable for all finite Reynolds numbers. Nagata's upper and lower solution branches appear subcritically at $\mathrm{Re}$ $=U_{w} h / \nu \approx 125$ from a saddle-node bifurcation, where $U_{w}$ is half the difference of the two wall velocities. In general, the upper-branch (or lower-branch) solutions generated from the bifurcation have a larger (or smaller) deviation from a laminar state. All the solutions have two spatial symmetries: $:^{1,21,22}$ a reflection with respect to the plane of $z=0$ plus a streamwise shift by $L_{x} / 2$,

$$
\left(u^{*}, v, w\right)\left(x, y^{*}, z\right)=\left(u^{*}, v,-w\right)\left(x+L_{x} / 2, y^{*},-z\right),
$$

and a rotation by $\pi$ around the line $x=y^{*}=0$ plus a spanwise shift by $L_{z} / 2$,

$$
\left(u^{*}, v, w\right)\left(x, y^{*}, z\right)=\left(-u^{*},-v, w\right)\left(-x,-y^{*}, z+L_{z} / 2\right) .
$$

The steady traveling-wave solution found by Waleffe ${ }^{4}$ for plane Poiseuille flow also has symmetry (5).

Figure 1 and Table II summarize the properties and the computational parameters of the solutions used below as representative of this flow. All the cases were computed using $16 \times 16$ complex Fourier coefficients in the wall-parallel directions and 33 Chebychev polynomials in $y$. This represents a resolution of at least 15, 9, and 5 wall units, respectively, in the $x, y$, and $z$ directions, which is better than most direct simulations of turbulence. ${ }^{18,23}$ The most marginal direction is $y$ and grid convergence was tested at $\mathrm{Re}=400$ by reducing the resolution to 16 polynomials. The changes in Fig. 1 were within the size of the symbols.

Note that both the upper and the lower branches have higher dimensionless shear rates at the wall, $1+\left(h / U_{w}\right) \mathrm{d} u_{0} /\left.\mathrm{d} y\right|_{y^{*}= \pm 1}$, than the unit shear of the laminar state. The range of existence of the solutions in Fig. 1 is always close to $L_{z}^{+}=100$, as in the observed mean separation 


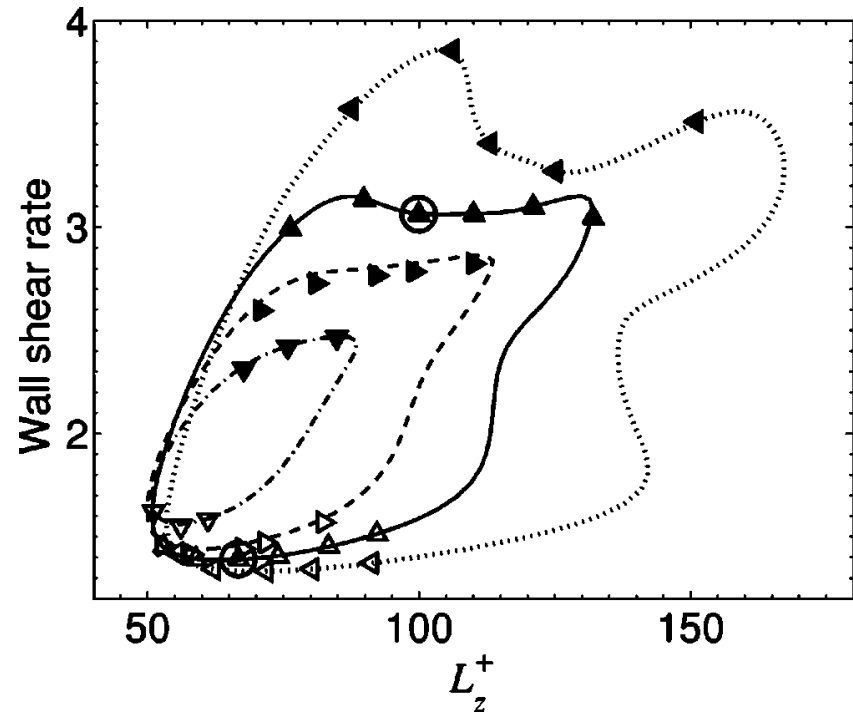

FIG. 1. Dimensionless wall shear rate for the solutions in Table II, vs the spanwise period $L_{z}^{+}$. $\nabla, \operatorname{Re}=200 ; \triangleright, 300 ; \triangle, 400 ; \triangleleft, 600$. In all cases $L_{x}$ $=2 \pi h$. The lines are drawn from the tracking algorithm, and the points distinguished by symbols are used later for a more detailed study. Solid symbols are classified as "upper branch," open ones as "lower branch." The two circled cases are those in Fig. 7.

of sublayer streaks. The limits of that range, $L_{z}^{+} \approx 50-150$, are also in good agreement with the range of streak spacings, $\lambda_{z}^{+} \approx 50-200$ found in real boundary layers. ${ }^{24}$

Two time-periodic solutions for a plane Couette flow are taken from Kawahara and Kida, ${ }^{10}$ using $\mathrm{Re}=400, L_{x}$ $=1.755 \pi h$, and $L_{z}=1.2 \pi h$, which are essentially the same as those of the minimal plane Couette turbulence studied in Ref. 25. The latter approximately satisfies the two symmetries (5) and (6) spontaneously, ${ }^{10,25,26}$ but they have been explicitly imposed on the time-periodic solutions. Their parameters are summarized in Table III.

Figure 2 shows the wall shear rate of Nagata's steady waves as a function of the streamwise wavelength, and also those of the two periodic solutions. There is no steady Nagata solution for the conditions of the periodic orbits, but the wall shear rate of the orbit $\mathrm{O} 1$ is roughly the same as that of Nagata's upper branch, while that of the orbit $\mathrm{O} 2$ is closer to

TABLE II. Approximate parameter ranges for the "lower branch" L and "upper branch" U Nagata solutions used in the paper. $h^{+}$is half the wall separation in wall units. All the cases use $L_{x}=2 \pi h$.

\begin{tabular}{lcccccc}
\hline \hline & $\operatorname{Re}$ & $h^{+}$ & $L_{z}^{+}$ & $u_{\max }^{\prime+}$ & $v_{\max }^{\prime+}$ & $\omega_{x, \max }^{\prime+}$ \\
\hline L1 & 200 & $18-18$ & $51-61$ & $2.59-3.19$ & $0.29-0.40$ & $0.12-0.12$ \\
L2 & 300 & $21-22$ & $53-82$ & $3.10-4.32$ & $0.21-0.30$ & $0.08-0.10$ \\
L3 & 400 & $24-25$ & $53-92$ & $3.20-5.09$ & $0.17-0.30$ & $0.07-0.08$ \\
L4 & 600 & $29-29$ & $55-91$ & $3.49-5.62$ & $0.14-0.28$ & $0.05-0.06$ \\
& & & & & & \\
U1 & 200 & $21-22$ & $68-85$ & $2.26-2.42$ & $0.61-0.70$ & $0.15-0.16$ \\
U2 & 300 & $28-29$ & $71-110$ & $2.46-2.68$ & $0.64-0.83$ & $0.13-0.13$ \\
U3 & 400 & $35-35$ & $76-132$ & $2.79-3.42$ & $0.53-0.89$ & $0.11-0.13$ \\
U4 & 600 & $44-48$ & $87-151$ & $3.15-3.61$ & $0.61-0.94$ & $0.09-0.11$ \\
\hline \hline
\end{tabular}

TABLE III. Parameters of the two periodic solutions in Ref. 10. $T_{p}^{+}$is the temporal period of the solution and the turbulence properties are averaged over each cycle. Both cycles are traversed clockwise in the representation in Figs. 5 and 11 and counterclockwise in the representation in Fig. 14.

\begin{tabular}{cccccccc}
\hline \hline & $L_{x}^{+}$ & $L_{z}^{+}$ & $h^{+}$ & $T_{p}^{+}$ & $u_{\max }^{\prime+}$ & $v_{\max }^{\prime+}$ & $\omega_{x, \max }^{\prime+}$ \\
\hline $\mathrm{O} 1$ & 190 & 130 & 34.4 & 188 & 3.18 & 0.741 & 0.125 \\
$\mathrm{O} 2$ & 154 & 105 & 27.9 & 299 & 4.62 & 0.231 & 0.084 \\
\hline \hline
\end{tabular}

Nagata's lower branch. Hereafter, the former solution will be referred to as the "upper" periodic solution, while the latter will be called the "lower" periodic solution, although there does not appear to be a continuous connection between the two. The upper cycle exhibits a full regeneration cycle of near-wall coherent structures, and approximates well the low-order turbulence statistics of minimal plane Couette flow (see Fig. 3). It is interesting to note that both the upper and lower branches of Nagata steady waves have minimum streamwise wavelengths which are relatively independent of the Reynolds number. In the case of the upper branch this minimum length is approximately consistent with the wavelength $\lambda_{x}^{+} \approx 250-300$ below which wall turbulence cannot be sustained. ${ }^{14,15}$

The solution loci in Fig. 2 are open towards long wavelengths. Given the number of modes that can be used in practice by our continuation algorithm, wavelengths longer than those in the figure would be numerically inaccurate. Solution curves closed towards large $L_{x}$ were found in Ref. 27 , and closed solutions (not shown) were also found by us for narrower boxes $\left(L_{x}=0.8 \pi\right)$. It may very well be that the curves in Fig. 2 close at some longer wavelength.

\section{CLASSIFICATION OF SOLUTIONS}

In this section, we look at the similarities and differences among the simple solutions available in the literature for Couette and Poiseuille flows, including the equilibrium and periodic solutions described in the preceding section. Figure

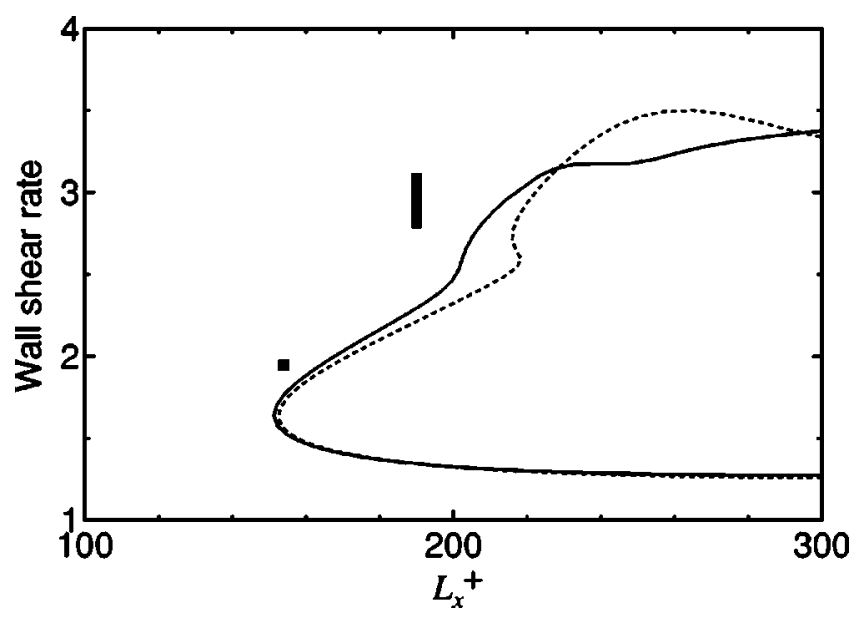

FIG. 2. Wall shear rate for the Nagata solutions vs the streamwise wavelength $L_{x}^{+}$. -, $\operatorname{Re}=400 . L_{z}=1.2 \pi h ;----, \operatorname{Re}=600 . L_{z}=\pi h$. The upper long (lower short) vertical thick segment represents the wall shear variation of the time-periodic solution $\mathrm{O} 1(\mathrm{O} 2)$ in Table III. 

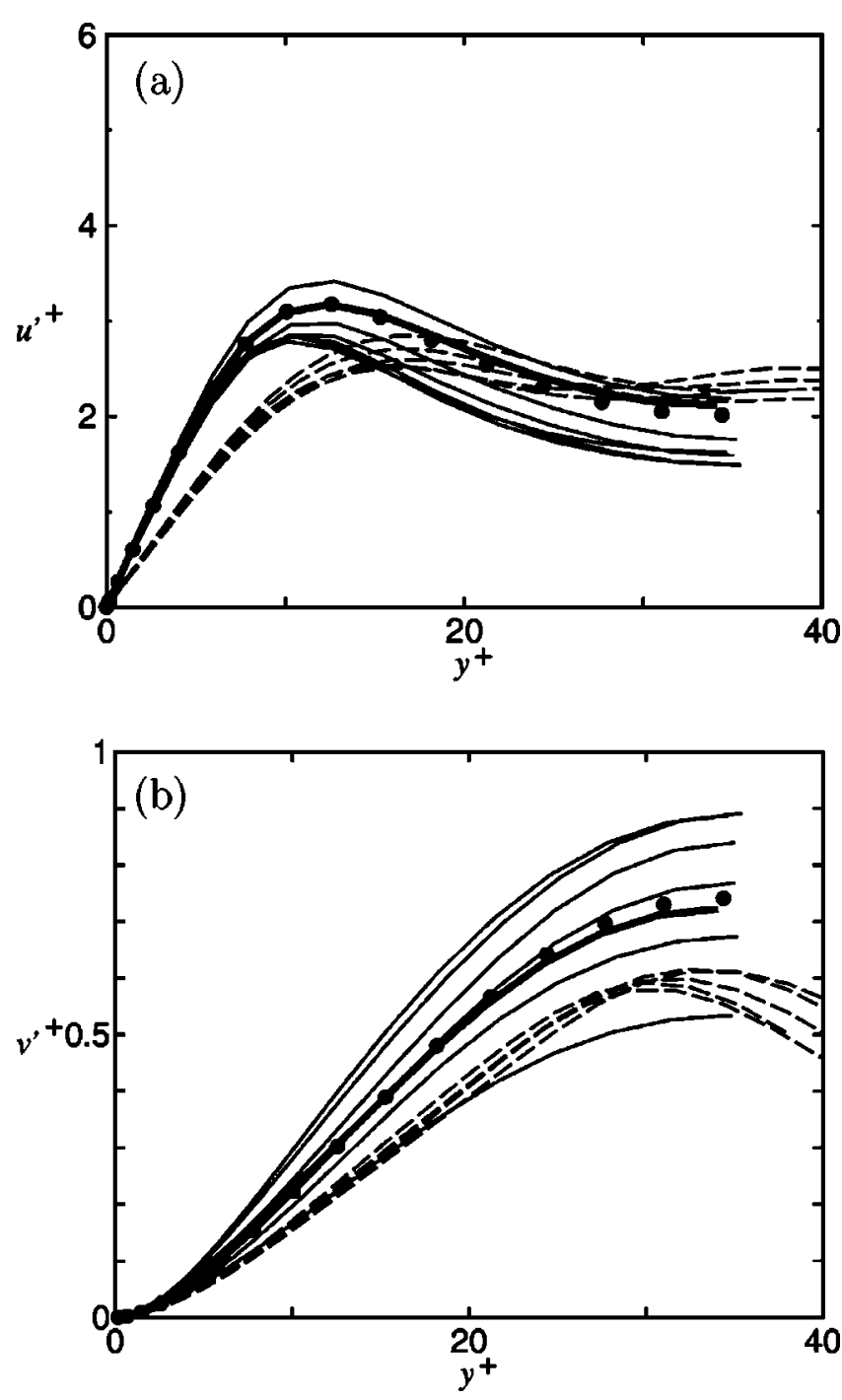

FIG. 3. r.m.s. velocity profiles for ----, the autonomous solutions from Table I; - , upper-branch solutions of case U3 in Table II; - "upper" cycle O1 in Table III. The heavy solid line is the minimal Couette simulation $\mathrm{C} 1$ in Table IV. (a) Streamwise component. (b) Wall-normal component.

3 shows the streamwise and wall-normal r.m.s. velocity profiles, $u^{\prime}$ and $v^{\prime}$, for the upper-branch Nagata equilibrium solutions, for the upper cycle of Ref. 10, and for the autonomous steady waves. The figure also includes the statistics of a fully-turbulent minimal Couette simulation. Figure 4 does the same for the lower-branch Nagata solutions and for the lower cycle. All the profiles in Fig. 3 agree roughly among themselves, as do those in Fig. 4, but the two families are very different from each other. The upper-branch solutions are very close to minimal near-wall turbulence, which is included in Fig. 3, and are characterized by a relatively strong wall-normal velocities, and by weaker streamwise fluctuations. The lower solutions have much weaker wall-normal velocities and stronger streamwise fluctuations. Since nearwall turbulence is known to be dominated by streamwisevelocity streaks and by quasistreamwise vortices, ${ }^{13}$ and since the latter are responsible for most of the generation of wallnormal velocity, the relative magnitudes of $v^{\prime}$ and of $u^{\prime}$ can respectively be used as indicators of the strength of the vor-
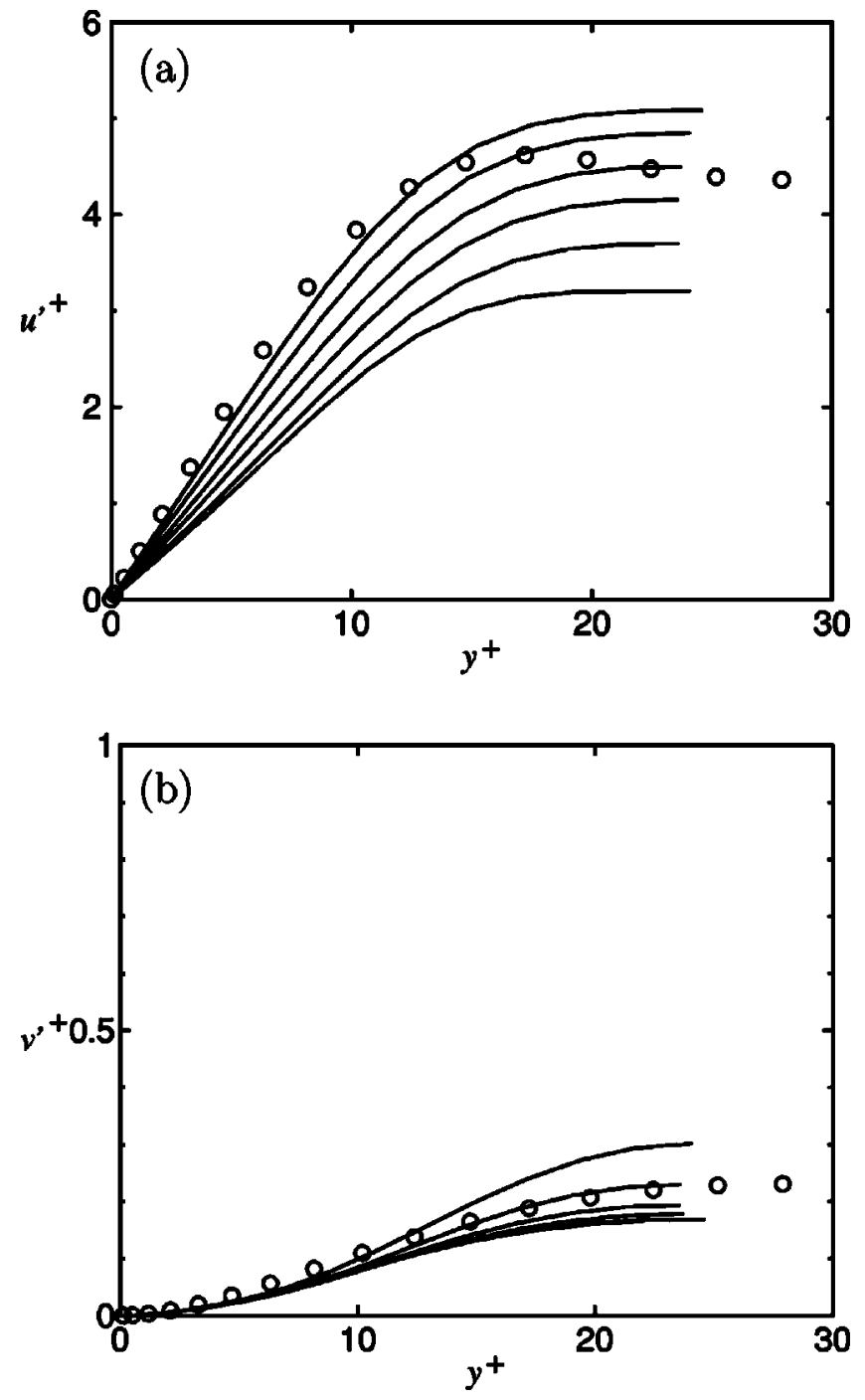

FIG. 4. r.m.s. velocity profiles for -, lower-branch solutions of case L3 in Table II; $\bigcirc$, "lower" cycle $\mathrm{O} 2$ in Table III. (a) Streamwise component. (b) Wall-normal component.

tices and of the streaks. ${ }^{17}$ We will therefore characterize the upper-branch solutions as "vortex-dominated," and the lower-branch ones as "streak-dominated."

The characteristics of the fluctuation profiles of the different solutions are summarized in Fig. 5, where each solution is represented by a single point whose coordinates are the maximum values, $u_{\max }^{\prime}$ and $v_{\max }^{\prime}$, of its intensity profiles. Most solutions fall into one of the two classes discussed above. In the upper-left corner of the plot we have the vortex-dominated solutions, and in the lower-right corner the streak-dominated ones. In the former class we find the three upper-branch families discussed above, and in the latter the two lower-branch Couette solutions. The unstable permanent wave obtained by Toh and Itano ${ }^{3}$ in a Poiseuille flow, and the heteroclinic connection identified by the same authors ${ }^{28}$ are streak-dominated solutions. The figure also includes the two permanent-wave solutions given by Waleffe ${ }^{2}$ for Poiseuille flow, which are classified as lower or upper branch according to the original reference, although they are too close to the turning point to differ too much from each other. All that can 


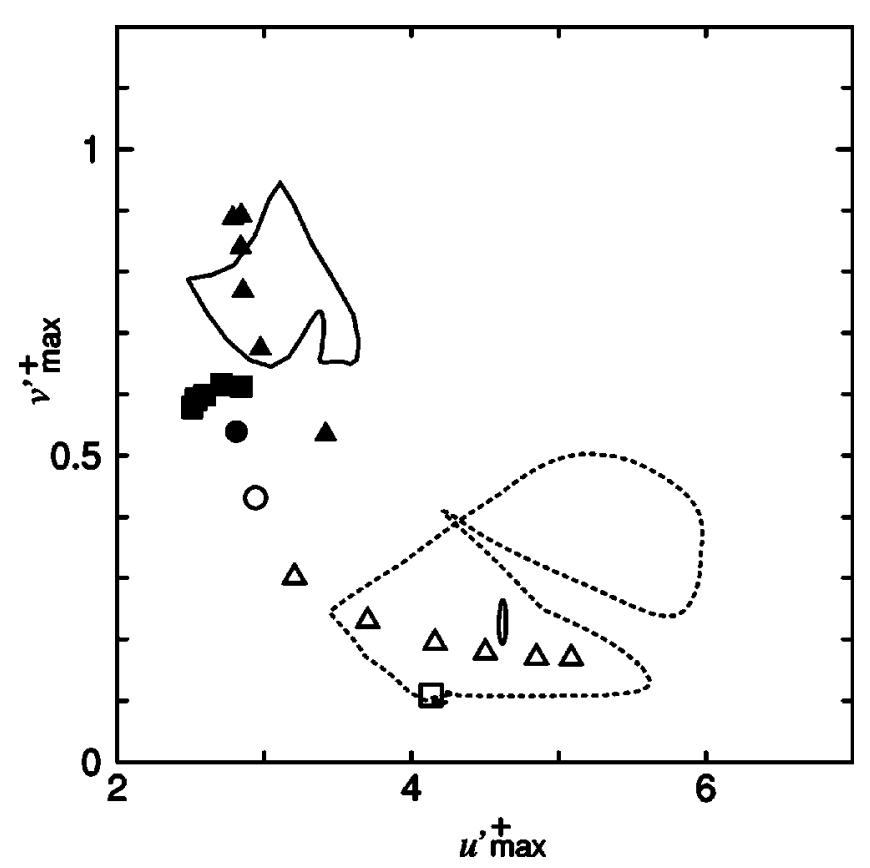

FIG. 5. Classification into upper and lower solutions in terms of the maximum streamwise and wall-normal r.m.s. velocities, $u_{\max }^{\prime}$ and $v_{\max }^{\prime}$. The solid large and small loops represent the upper and the lower periodic solutions $\mathrm{O} 1$ and $\mathrm{O} 2$ in Table III. $\triangle$, Nagata steady solution L3-U3 from Table II. Solid symbols are classified as upper branch, and open ones as lower branch; $\mathbf{\square}$, autonomous solutions from Table I. Other traveling-wave solutions for plane Poiseuille flow are also shown for comparison; $\bullet$, Waleffe (Ref. 2) upper-branch solution for $Q / \nu=1303$, where $Q$ is the volume flux per unit span. $L_{x}^{+} \times L_{z}^{+} \times h^{+}=387 \times 149 \times 123$; $\bigcirc$, Waleffe (Ref. 2) lowerbranch solution for $Q / \nu=1390, L_{x}^{+} \times L_{z}^{+} \times h^{+}=379 \times 146 \times 121$; $\square$, Toh and Itano (Ref. 3) asymmetric wave for $Q / \nu=4000, L_{x}=\pi$, and $L_{z}=0.4 \pi$. The dotted loop is the periodiclike solution in Ref. 28.

be said about them is that they are ordered in the right way in this representation with respect to the classification used for the other solutions.

It is remarkable that two families are enough to classify all the solutions discussed here, which include Poiseuille, autonomous, and Couette flows, and steady, traveling-wave, and temporally periodic solutions computed by a variety of techniques. The homogeneity within each class is indeed only approximate. It is clear from Fig. 3 that, while the maxima of $u^{\prime}$ is almost the same in the autonomous and in the Couette flows, the peaks are located farther from the wall in the former than in the latter. It is also clear in Fig. 5 that $v^{\prime}$ tends to be stronger in Couette flows than either in the autonomous or in the Poiseuille solutions.

The classification into two families is on the other hand relatively independent of the parameters of the solutions. Figure 6 shows several Nagata equilibrium solutions obtained by varying $L_{z}$ for a fixed $L_{x}$, using different Reynolds numbers. The separation into two families persists even when the Reynolds number is increased by a factor of 3 , and a similar result (not shown) is obtained when the box length is increased by $50 \%$.

Similar classifications can be obtained using other variables, but not all of them are as clear as the previous one. Consider, for example, the substitution of $v_{\max }^{\prime}$ by the maximum $\omega_{x, \max }^{\prime}$ of the intensity profile of the streamwise vortic-
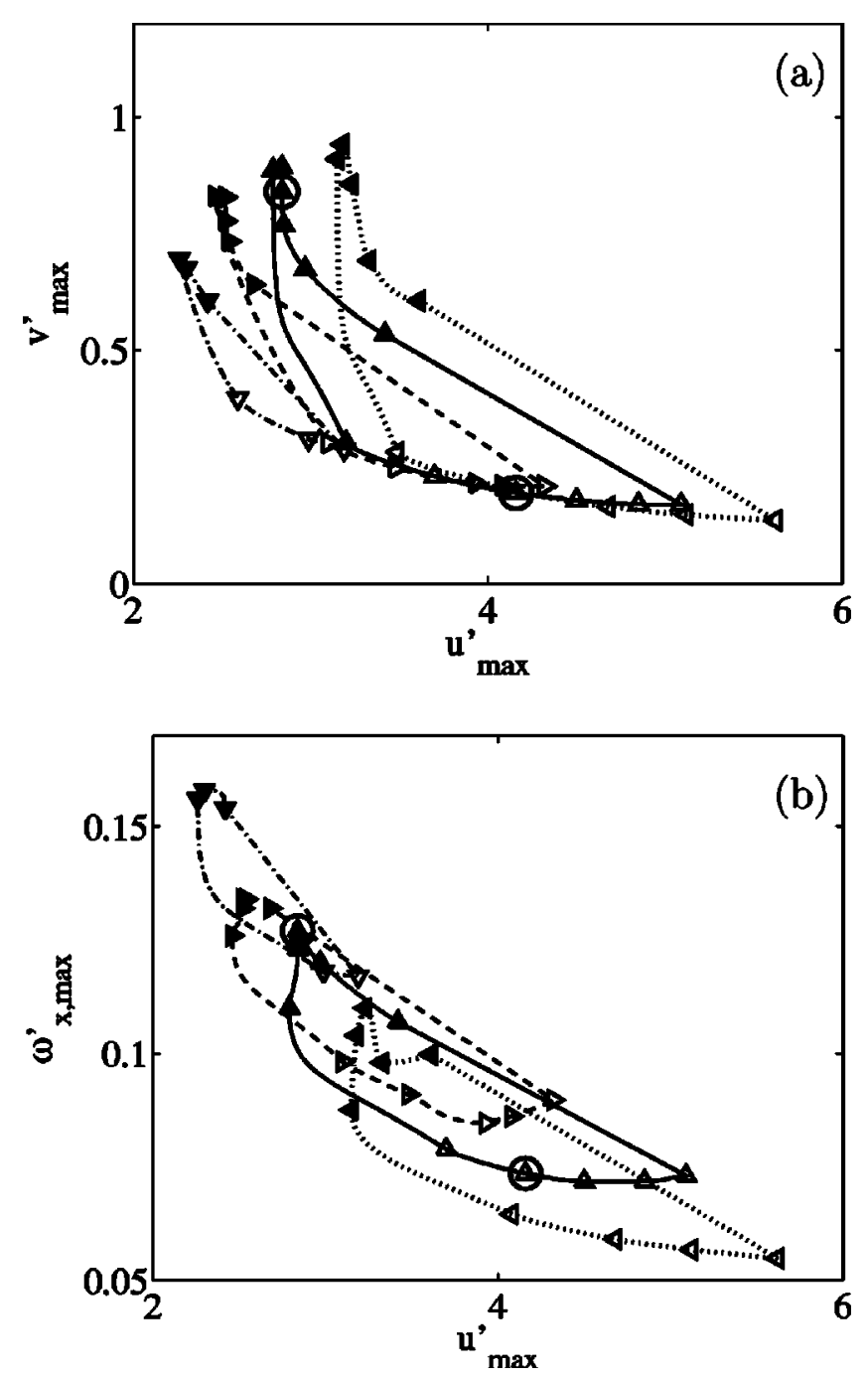

FIG. 6. Peak intensities for all the Nagata solutions in Table II. (a) $u_{\max }^{\prime}-v_{\max }^{\prime}$. (b) $u_{\max }^{\prime}-\omega_{x, \max }^{\prime}$. Symbols are as in Fig. 1. The lines are drawn to aid the eye. Only the symbols correspond to actual data.

ity, which could be considered a more direct indicator of the strength of the streamwise vortices. The maximum of interest for us is the one around $y^{+} \approx 15-20$, which in fully developed turbulence corresponds to the quasistreamwise vortices. ${ }^{18}$ There is usually a second maximum of $\omega_{x}^{\prime}$ at the wall itself, which is related to the interactions of the vortices with the transverse no-slip condition, and sometimes one more near the center of the flow, which is associated with the outer structures. Occasionally, specially at very low Reynolds numbers, some of those maxima merge, and it is impossible to define a maximum associated with the streamwise vortices. The vorticity maxima for the Nagata solutions are displayed in Fig. 6(b). It is seen, for example, that, while the maxima of $u^{\prime}$ and $v^{\prime}$ increase with Reynolds number for the upper Nagata solutions, those of $\omega_{x}^{\prime}$ decrease. By studying individual profiles it is seen that the reason is that the vortex and wall peaks tend to merge at very low Reynolds numbers, but the consequence is that the classification in terms of $\omega_{x}^{\prime}$ is less informative than that in terms $v^{\prime}$. Moreover, even if the vortices are the primary reason for the wallnormal velocity, it is the velocity itself that is dynamically 

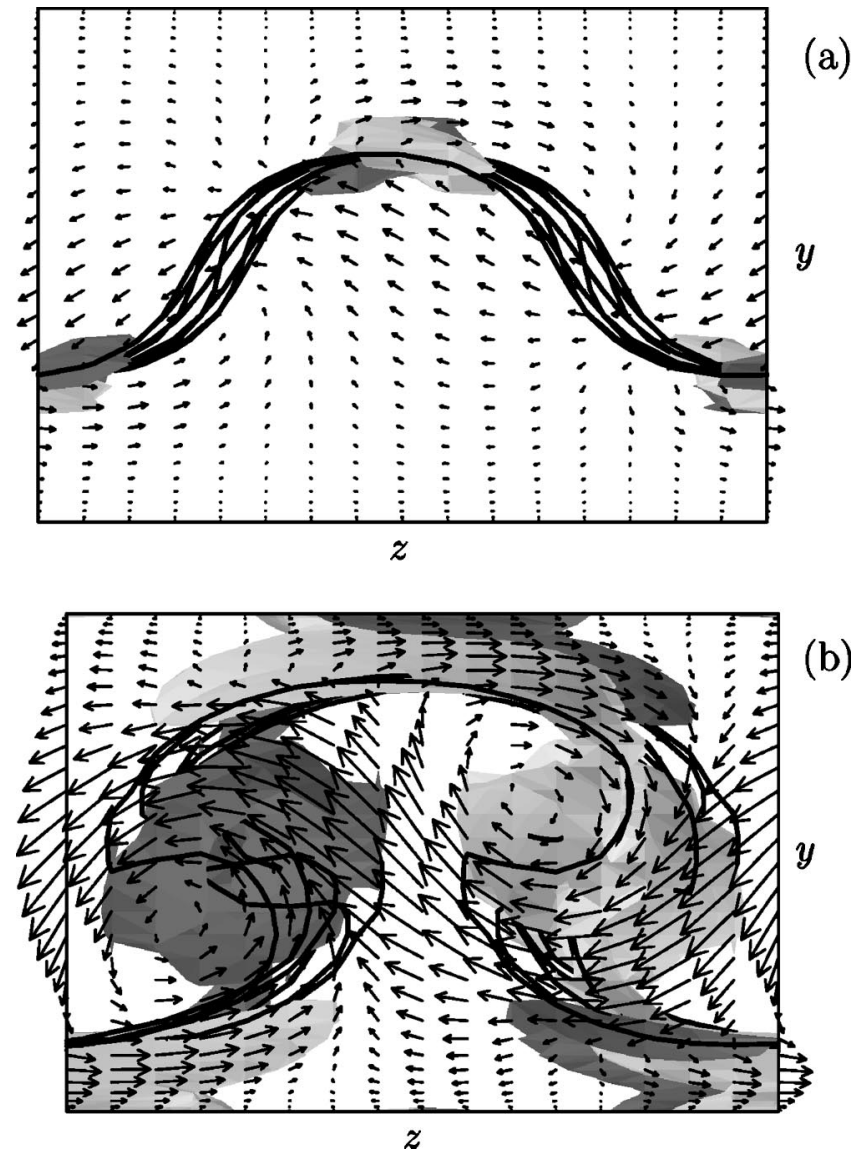

FIG. 7. Projection of the $\omega_{x}$ and velocity fields on the cross-plane $(z-y)$, for the solutions in Table II. $\operatorname{Re}=400, L_{x} \times L_{z}=2 \pi h \times 0.9 \pi h$. The thick solid lines are different sections of the surface $u=0$. Arrows are the cross-plane velocities at $x=0$, which is defined so that the first streamwise harmonic of $u$ is proportional to $\cos \left(2 \pi x / L_{x}\right)$. The arrows are uniformly scaled in wall units, and the longest arrows in (b) are roughly $1.9 u_{\tau}$. The dark- and lightgray objects are isosurfaces $\omega_{x}^{+}= \pm 0.155$. The two solutions used here are circled in Figs. 1 and 6. (a) Lower branch, $L_{z}^{+}=67$. (b) Upper branch, $L_{z}^{+}=99$.

significant, by advecting the mean shear to generate the streaks. From now on the variable pair $\left(u_{\max }^{\prime}-v_{\max }^{\prime}\right)$ will be our primary tool for comparing flows, although $v_{\max }^{\prime}$ will still be occasionally referred to as the "vortex intensity."

\section{A. The structure of the flow field}

Besides the statistical differences just discussed between the upper and lower branches, there are significant differences in their spatial structures. Consider the two flow fields in Fig. 7, which correspond to lower and upper Nagata solutions at comparable Reynolds numbers and dimensions. They differ mainly in the location of the streamwise vorticity, which is concentrated in the form of sheets in the case of the lower branch, but which also has two concentrated tubular vortices in each flank of the streak in the case of the upper branch. In both cases the vorticity structures are staggered streamwise, and the streak itself is deformed sinusoidally. Three-dimensional representations of these flows, especially of the upper branch, can be found in the original papers describing these solutions. ${ }^{2,5,10}$

It has been noted elsewhere that the lower-branch solu- tions are very similar to the linearly unstable eigenfunctions of the sinusoidal mode of a straight streak. ${ }^{7}$ This makes sense because the lower-branch saddle in Ref. 3 was found by tracing its stable manifold from some unstable streaky flow. Those eigenfunctions contain little streamwise vorticity in the flanks of the streak, and do not include vortices with circular cores, which are typically only formed later by the nonlinear rollup of vortex sheets.

The origin of the instability and of the streamwise vorticity can be understood by considering its evolution equation,

$$
\left(\frac{\mathrm{D}}{\mathrm{D} t}-\nu \nabla^{2}\right) \omega_{x}=\partial_{x} v \partial_{z} u-\partial_{x} w \partial_{y} u+\omega_{x} \partial_{x} u .
$$

For a straight streak without streamwise vorticity both $\partial_{x}$ and $\omega_{x}$ vanish, and so do the three source terms in the right-hand side of (7). The first two source terms are linear in $\partial_{x}$. If the streak is kinked slightly, they create streamwise vorticity by reorienting the shear around the streak into a streamwise vortex sheet. In a sinusoidal mode, this streamwise vorticity changes sign along $x$ and further deforms the streak by creating a spanwise velocity which varies with $x$. This is the feedback mechanism of the sinuous instability responsible for the lower branch.

It is tempting to blame the third term in the right-hand side of (7) for the collapse of the vortex sheets into tubes as the intensity of the kinking increases. This term is bilinear in $\partial_{x}$ and $\omega_{x}$, and represents the stretching of $\omega_{x}$ by the streamwise variation of $u$. The strain $\partial_{x} u$ is positive whenever the kinked low-velocity streak follows higher-velocity fluid, and is highest towards the middle of its vertical walls. It is here that the initial streamwise vortex sheet is strained most, and where it can collapse into tubular vortices. ${ }^{29}$ On the other hand it is known that two-dimensional vortex sheets, which are not stretched, roll into circular cores in times of the order of the inverse of their vorticity, which are in general shorter in wall-bounded flows than the time needed for them to build a streak. ${ }^{30}$ The criterion for rollup is that the internal Reynolds number of the streak should be large enough for advection to overcome viscous diffusion. The upper branch would then appear as an equilibrium solution in which the sheets of streamwise vorticity created by the instability have become intense enough to roll into cores. Stretching helps to localize them along the streak.

We have seen that both types of solutions exist, with and without collapsed vortices, arising from a saddle-node bifurcation at some onset Reynolds number. It is however clear that both configurations are unstable. The $x$ dependent $\omega_{x}$ distribution kinks the streak, which generates further streamwise vorticity, and further kinking. This eventually weakens the streak by mixing the velocity gradient along its flanks, and consequently the vortices created by that gradient.

Note that there is no one-to-one correspondence between the strength of the vortices and that of the streak. The latter is the consequence of the action of the vortices over a period of time, and this time scale is as important in determining the streak intensity as the vortex strength. There is little doubt that, in the absence of viscosity or of other limiting factor, a 


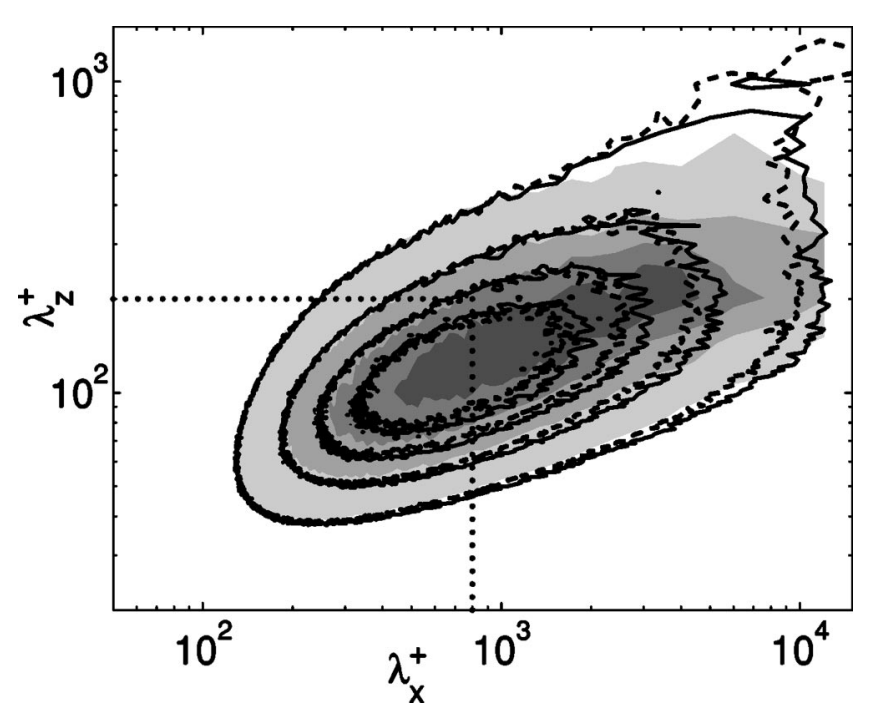

FIG. 8. Premultiplied spectra of the streamwise velocity component $k_{x} k_{z} E_{u u}$ as a function of the spanwise and streamwise wavelengths $\lambda=2 \pi / k \cdot y^{+}$ $=16$. Shaded, autonomous flow in a large box (Ref. 17), masked above $\delta_{1}^{+}$ $=60$; - - full channel (Ref. 33) at $h^{+}=550$; ----, full channel (Ref. 34) at $h^{+}=950$. The dotted rectangle is the first zero of the transfer function for a convolution box of size $b_{x}^{+} \times b_{z}^{+}=400 \times 100$.

permanent vortex will eventually pump the mean velocity profile into uniform streamwise regions in which the streamwise velocity is that of one or of the other wall. Viscosity or instability limit that distortion, and the simplest explanation of the weaker streaks measured for the upper-branch solutions is that the flank vortices shorten the effective time scale of the pumping by providing an effective eddy viscosity that homogenizes the streamwise-velocity profile. An equivalent explanation closer to the language of turbulence is that the "overhangs" of the streak in Fig. 7(b) are first- and thirdquadrant events ${ }^{31,32}$ which substract from the production of turbulent energy.

\section{COMPARISON WITH TURBULENT FLOWS}

\section{A. Flow type and Reynolds-number effects}

We will compare in this section the previously discussed simple solutions to the Navier-Stokes equations with fully developed turbulence in large computational boxes, but some discussion is first needed on what is being compared, and of the differences between different flows and between different Reynolds numbers.

It should be clear that we may only expect the solutions in the preceding section to be related to structures in the buffer and viscous sublayers of fully turbulent flows, but it is also true that not all the near-wall structures can be modeled in this way. Consider the spectra of the streamwise velocity displayed in Fig. 8, which includes an autonomous flow with "no" outer layer, ${ }^{17}$ and two full channels at different Reynolds numbers. ${ }^{33,34}$ The spectra are scaled in wall units and have three regions. In the lower-left corner, corresponding to "minimal" structures of sizes comparable to the solutions discussed above, the spectra agree well. Farther to the right, at scales which are too long and too wide to be compared with the simple solutions, there are spectral "tails" which also agree among themselves, although with different maximum lengths. It was shown in Ref. 17 that these tails are passive in the sense that they can be removed without disturbing the minimal scales, and that they can be explained as wakes of the smaller structures. The last part of the spectrum is the upper-right corner, which differs among the three flows. Those structures scale in outer units, contain no Reynolds stresses at this wall distance, and extend deep into the outer flow. ${ }^{33}$ The only spectral range that can be compared to the simple solutions discussed above is, therefore, the lowerleft corner, but that range is specially important because it contains the structures that are both active in the sense of participating in the generation of turbulent energy in the buffer layer, and autonomous in the sense of requiring neither larger or taller scales to survive. They form the "nonlinear core" of buffer-layer turbulence.

The behavior of the turbulence statistics with the Reynolds number is connected with this spectral classification. The total energy $u^{\prime 2}$ of the streamwise velocity fluctuations is the integral of the spectrum, and it follows from Fig. 8 that, if we consider all the structures in the buffer layer, $u^{\prime}$ should depend on $h^{+}$through the dependence of the spectrum. ${ }^{35}$ If on the other hand we only consider structures within the minimal dotted box in the figure, the partial $u^{\prime}$ should become independent of the Reynolds number.

This is a particular case of the dependence of the turbulence statistics on the size of the averaging box, which means that boxes of different sizes cannot be directly compared, even within the same flow, because individual small boxes are not statistically "converged." In particular, the fluctuation profiles of the simple solutions are not comparable to the intensity profiles compiled in experiments or in computations. To allow the comparison in our case, each wall of the large boxes is divided into sub-boxes of minimal size $b_{x}^{+}$ $\times b_{z}^{+} \approx 380 \times 110$, and the statistics are compiled over them. In addition, each wall is treated independently, and the intensity profiles are compiled only from the wall to the central plane. Each sub-box is characterized by its maximum r.m.s. intensities, and the values for different sub-boxes are summarized as a joint probability density function of the two quantities, compiled over all the sub-boxes and over time. Each flow is therefore not characterized by a single point, but by the probability distribution of the possible states of a subbox of a given size. Although the effect of this procedure on the spectral space is not straightforward, its main effect is to subtract from the velocity its local average, and can be approximated as windowing the spectrum with one minus the Fourier transform of the averaging box, which decreases rapidly above $\lambda=2 b$. This spectral window is represented by dotted lines in Fig. 8, and contains all the active autonomous scales discussed above.

The effect of this local averaging procedure is shown in Fig. 9(a), which displays the maximum of the r.m.s. velocity fluctuations for several turbulent flows as a function of the Reynolds number. As predicted above, $u_{\max }^{\prime}$ increases with $h^{+}$, but when the same flows are analyzed over boxes of minimal size, as in the closed symbols in the figure, that effect disappears. Another example is shown in Fig. 9(b), which shows the probability distributions of the maximum 

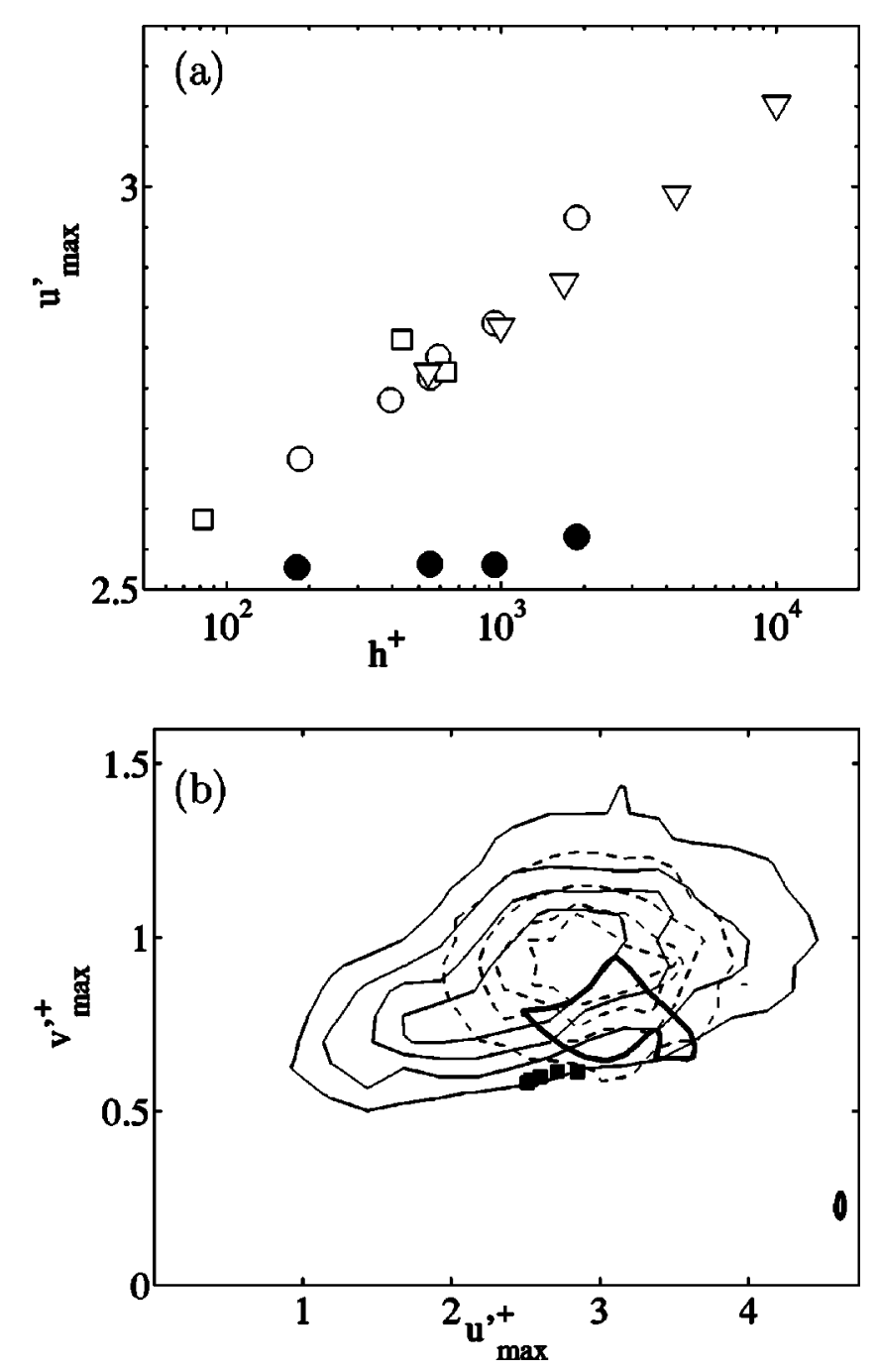

FIG. 9. (a) Maximum of $u^{\prime}$. Open symbols are either full simulations or experiments. Solid symbols are computed over averaging boxes of size $400 \times 100$. $\bigcirc$, Poiseuille (Refs. 33 and 34); $\nabla$, boundary layers (Ref. 35); $\square$, Couette (Refs. 36 and 38). (b) Comparison between the joint p.d.f.s of the maximum fluctuation intensities in the minimal Poiseuille flow P4 in Table $\mathrm{IV}$, depending on the width of the averaging box.,$- b_{z}^{+}=110 ;---, b_{z}^{+}$ $=220$. The probability contours contain $30 \%, 50 \%, 70 \%$, and $90 \%$ of the data. The solid squares are the permanent waves from Table I, and the left and right heavy solid lines are, respectively, cycles $\mathrm{O} 1$ and $\mathrm{O} 2$ from Table III.

intensities for two identical minimal flows, compiled over boxes which are approximately twice wider in one case than in the other. The statistics in the wider box are more concentrated than those in the narrower one, and most of the "wings" of the latter are missing in the former.

The next question is whether different flows can be compared to one another. There are well-documented differences between the near-wall behavior of Poiseuille and Couette turbulent flows at low Reynolds numbers, ${ }^{36}$ which are presumably related to the discrepancies between the corresponding elementary solutions, but they disappear near the wall as the Reynolds number increases. The $u^{\prime}$ maxima in Fig. 9(a), which are in the near-wall region, fall in the same line for all the flows included. Another example is the wall-normal velocity of the autonomous simulations, which is lower than in Poiseuille flow, because $v^{\prime}$ peaks away from the wall and is

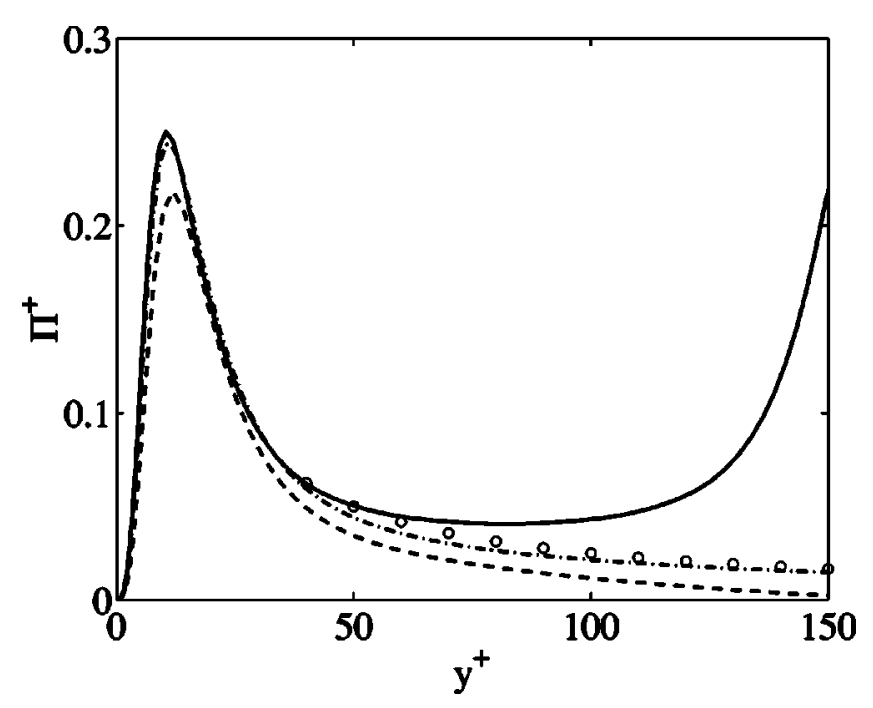

FIG. 10. Production profiles from full-size simulations. - Couette flow (Ref. 36) at $h^{+}=82$; ----, Poiseuille (Ref. 18), $h^{+}=180$; ---, Poiseuille (Ref. 34), $h^{+}=1880$; $\bigcirc$, asymptotic infinite-Reynolds number distribution from the logarithmic profile with Kármán constant $\kappa=0.4$.

damped by the numerical mask. Even in that case, when $v^{\prime}$ is measured at a given $y^{+}$near the wall, the fluctuations of both flows agree well. ${ }^{5,17}$ The wall-normal velocities of Poiseuille flow also vary at low Reynolds numbers, ${ }^{23,37}$ and $v_{\max }^{\prime+}$ increases from $\approx 0.85$ to 1.08 when $h^{+}$increases from 180 to 1000. Jiménez and Pinelli ${ }^{15}$ found the same effect in minimal channels with $h^{+} \leq 600$, but experiments in boundary layers at much higher Reynolds numbers ${ }^{35}$ suggest that the growth of the wall-normal-velocity peak saturates when $h^{+} \gtrsim 1000$. There is no comparable effect for Couette flow, except perhaps at very low Reynolds numbers, and the few available Couette experiments at moderate to high Reynolds numbers ${ }^{38,39}$ show that $v_{\max }^{\prime+} \approx 1$ in the range $h^{+}=50-800$. The spectra of $v^{\prime}$ are essentially contained within the minimal box of Fig. 8, and lacks both the Reynolds-number dependent wakes, or the outer structures of $E_{u u}{ }^{33}$ All the available evidence suggests that the structure of the near-wall layer is approximately universal when the Reynolds number is large enough.

The main reason for the differences of low-Reynolds number behavior of Poiseuille and Couette flows is probably their different mean velocity profiles, which is monotonic in the latter but not in the former. The production of turbulent kinetic energy is proportional to the gradient of the mean velocity, and the fluctuations in Poiseuille flow begin to be damped as soon as they cross into the opposite side of the channel. The same is true for the autonomous flows, in which the eddies which move into the numerical mask are immediately damped, but not in the Couette case, in which the monotonic velocity profile means that an eddy can cross almost to the opposite wall without being damped. This is clearly seen in Fig. 10, which shows profiles of the turbulent energy production, $\Pi=-\langle\tilde{u} \widetilde{v}\rangle \partial_{y} U$, where $\widetilde{u}$ and $\widetilde{v}$ are fluctuations with respect to the long-term mean velocity profile $U$, for a fully developed Couette flow at $h^{+}=82$, and for two Poiseuille flows at substantially higher Reynolds numbers. If we assume ${ }^{15}$ that the near-wall structures are governed by the 

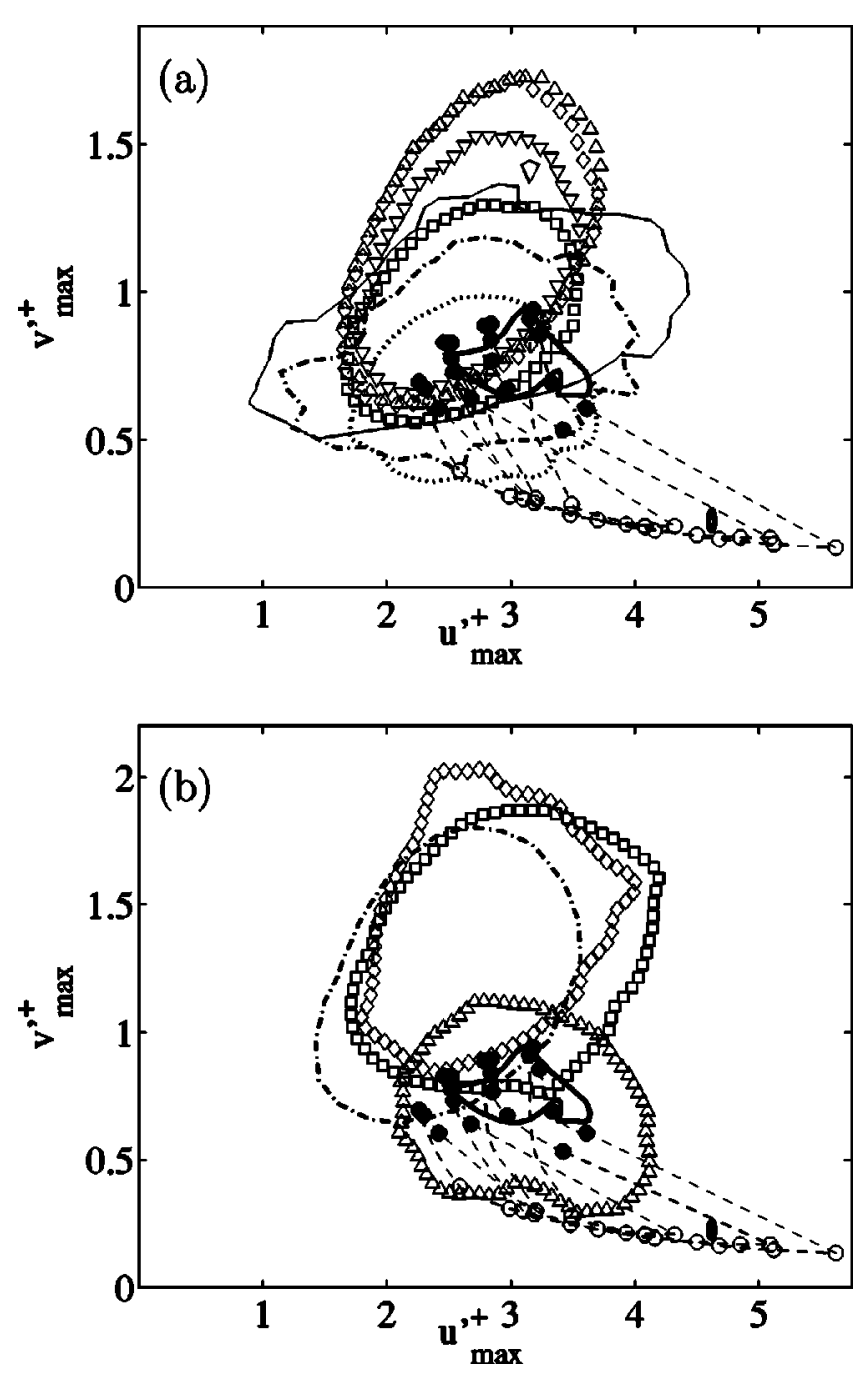

FIG. 11. Comparison between the joint p.d.f.s of the maximum fluctuation intensities $\left(u_{\max }^{\prime}-v_{\max }^{\prime}\right)$ in minimal and full turbulent flows and in simple solutions. Probability isolines contain $90 \%$ of data. The heavy solid lines are as in Fig. 9(b). The dashed lines with open and solid circles are Nagata's Couette solutions, as in Fig. 6(a). (a) Turbulent Poiseuille flows. Statistics are computed over boxes $b_{x}^{+} \times b_{z}^{+} \approx 380 \times 110$, and only those boxes for which the maximum is below $y^{+}=50$ are used. Open symbols are large-box simulations (Refs. 33 and 34). $\triangle, h^{+}=1880 ; \diamond, 950 ; \nabla, 550 ; \square, 180$. Lines are minimal-box Poiseuille simulations from Table IV. - , P4, $h^{+}=197 ;---$, $\mathrm{P} 2, h^{+}=123 ; \cdots \cdots . ., \mathrm{P} 1, h^{+}=85$. (b) Turbulent Couette flows. Simulations were run in boxes $L_{x}^{+} \times L_{z}^{+} \approx 380 \times 180$, but the statistics were computed over $b_{x}^{+} \times b_{z}^{+} \approx 380 \times 100$, over the full half-height of the flow. Open symbols are minimal-box Couette simulations from Table IV. $\triangle, \mathrm{C} 1, h^{+}=34$; $\square, \mathrm{C} 2$, $h^{+}=107 ; \diamond, \mathrm{C} 3, h^{+}=140$. ---, Full Poiseuille simulation at $h^{+}=950$, from (a), included to facilitate comparison.

region below $y^{+} \leq 60-100$, it is clear from the figure that the production in that layer is closer to the infinite-Reynoldsnumber limit for the low-Reynolds-number Couette flow than for any of the Poiseuille flows. The near-wall layer of Couette flows can then be "fully developed" at Reynolds numbers as low as $h^{+}=50-80$, in agreement with the experimental evidence mentioned above.

\section{B. Comparisons with full turbulence}

Consider first the solutions in the Poiseuille family. As explained above, to compare the simple solutions with large
TABLE IV. Parameters of the minimal Poiseuille $\mathrm{P}$ and Couette $\mathrm{C}$ flows used in the figures in Secs. IV and V. $L_{x}$ and $L_{z}$ are the dimensions of the computational box, while $b_{x}$ and $b_{z}$ are the dimensions of the sub-boxes over which statistics are compiled. Re for the Poiseuille cases is based in the bulk velocity and the half height. For the Couette case it is based on half the velocity difference and the half height.

\begin{tabular}{ccccccc}
\hline \hline & $\operatorname{Re}$ & $L_{x}^{+}$ & $L_{z}^{+}$ & $h^{+}$ & $b_{x}^{+}$ & $b_{z}^{+}$ \\
\hline P1 & 1170 & 410 & 180 & 85 & 410 & 110 \\
P2 & 1800 & 385 & 220 & 123 & 385 & 110 \\
P3 & 2925 & 450 & 125 & 181 & 450 & 125 \\
P4 & 2925 & 375 & 220 & 197 & 375 & 110 \\
P5 & 30500 & 440 & 110 & 850 & 440 & 110 \\
P6 & 77200 & 450 & 110 & 1720 & 450 & 110 \\
& & & & & & \\
C1 & 400 & 188 & 128 & 34 & 188 & 100 \\
C2 & 2000 & 337 & 101 & 107 & 337 & 101 \\
C3 & 3000 & 440 & 106 & 140 & 440 & 106 \\
\hline \hline
\end{tabular}

turbulent channels, ${ }^{33,34}$ which have computational boxes of the order of $L_{x}^{+} \times L_{z}^{+} \approx 10000 \times 5000$, we compile for the latter the probability distributions of $u_{\max }^{\prime}$ and $v_{\max }^{\prime}$ computed over "minimal" sub-boxes of the same wall-parallel dimensions as the computational boxes of the former.

Figure 11(a) shows probability isolines containing $90 \%$ of the samples for each of the large flows, compared with the single points characterizing the instantaneous values of some of the simple solutions. The figure also includes p.d.f.s from fully turbulent minimal Poiseuille flows, which were computed for the present paper and which are summarized in Table IV. They include one case at $h^{+} \approx 190$ which should be similar to the full channel with the lowest-Reynolds number. The statistics for these minimal flows are computed in the same way as for the full channels, using sub-boxes of the size given in the table, independently of the size of their computational box. The numerical code used for the minimal Poiseuille simulations is the one in Ref. 14, and that used for the minimal Couette cases discussed below is the one from Ref. 40.

The p.d.f.s of the turbulent flows converge towards those of the upper-branch simple waves as the Reynolds number decreases. This is significant because the lowest Reynolds numbers used for the Poiseuille and Couette flows in Figs. 11(a) and 11(b), are very close to the minimum value for which turbulence can be sustained.

There is a trend for $v_{\max }^{\prime}$ to strengthen as the Reynolds number increases, but it saturates at large Reynolds numbers, as previously discussed. Note, in particular, that there is so little difference between the p.d.f.s at $h^{+}=950$ and $h^{+}$ $=1880$ in Fig. 11(a) that it is difficult to tell them apart. It is also significant that the lower limits of all the p.d.f.s reach down to the location of the permanent solutions, suggesting that the latter represent quiescent turbulent structures common to all Reynolds numbers, while the higher parts of the p.d.f. contain their "excited" states.

The difference between the minimal and the full channels in Fig. 11(a) is interesting, because their statistics are 
compiled over identical averaging boxes. Comparing the two cases with $h^{+} \approx 190$, it is clear that the minimal channel varies more, specially in the streak intensity $u_{\max }^{\prime}$. The same "wings" of the p.d.f. are found in all the minimal boxes, but not in the full ones. The left-hand wing represents occasions in which both the streaks and the vortices are weak, and the flow is close to local laminarization. Transient laminarization of one wall in minimal flows was already observed in Ref. 14 , and inspection of the temporal histories of the present minimal simulations (not shown) shows that the left-hand wings of the p.d.f.s correspond to events of this type.

The right-hand wings of the p.d.f.s represent strong streaks with weak vortices, and are conceptually similar to the lower-branch solutions discussed above, although Fig. 11(a) makes clear that the minimal Poiseuille flows always have stronger vortices than the equilibrium states belonging to that branch. Kawahara and $\mathrm{Kida}^{10}$ found that minimal Couette flow occasionally visits lower-branch solutions in which the streak grows for a while to very high intensities before breaking into a strong turbulent burst. We will refer to these strong streaks from now on as "metastable," because the evidence in the above reference suggests that the flow is not able to sustain such strong streak intensities for long times. The wider boxes in the present simulations do not visit this lower-branch cycle often enough to show in the p.d.f.s but it is tempting to interpret their right-hand wings as parts of the same process.

Neither of those two processes seems to happen in the full flows, although the tilt of their p.d.f.s towards the lowerleft corner of Fig. 11(a) suggests that some local laminarization occasionally occurs. The simplest interpretation is that the perturbations induced by the rest of the flow are enough to prevent individual streaks from either dying completely or from growing to metastable strengths.

We can also interpret in this light the differences between the two p.d.f.s in Fig. 9(b), in which the left-hand wing is missing from the wider box. Since we know from the p.d.f. computed over the narrower box that local laminarization occurs in this flow, its absence from the wider statistics shows that the two streaks that exist in the computational box never laminarize at the same time. This may be important in maintaining the flow, since the active streak can act as a trigger to revive the decaying one, and it is probably the reason why it was found in Ref. 5 that minimal turbulence at very low Reynolds numbers $\left(h^{+} \lesssim 100\right)$ could only be sustained in comparatively wide boxes of the order of $L_{z}^{+}$ $\approx 200$. Narrower boxes at such low Reynolds numbers sustain turbulence for fairly long times, but they eventually laminarize and do not recover. At somewhat higher Reynolds numbers both walls interact loosely, and one wall retriggers the other, but that mechanism eventually also fails. At very high Reynolds numbers the two walls interact little, and narrow boxes also occasionally die. Several of the boxes in Table IV had to be restarted at some point from slightly different initial conditions after they died, in some cases after running for $t^{+} \approx 5 \times 10^{4}$. In wider boxes one of the streaks in each wall can revive the whole flow, and extreme examples of this mutual triggering are the large autonomous boxes, ${ }^{15,17}$ in which essentially normal turbulence is maintained even when the wall-normal dimension of the flow is made as low as $\delta_{1}^{+} \approx 40$.

In Couette flows there are few large-box turbulent simulations to use as comparisons, but three minimal boxes were run for the present paper. Their parameters are given in Table IV, and their p.d.f.s are shown in Fig. 11(b). The lowest Reynolds number agrees very well with the statistics of the upper-branch Nagata solutions, as we had already seen in Fig. 3. Since those solutions correspond to the same flow at similar Reynolds numbers, it is not surprising that their agreement should be better than with the Poiseuille flows. As the Reynolds number increases, the Couette and Poiseuille statistics converge to one another, as discussed in the preceding section. Note that both in Figs. 11(a) and 11(b) the lowerbranch Nagata solutions are in a region of the parameter plane that is very different from the turbulent statistics. The Couette flows also have short "laminar" tails towards its lower-left-hand corner, and local excursions towards local laminarization can be seen in its history, but the effect is weaker than in the Poiseuille case. In contrast, its right-hand tail is stronger than in channels, and approaches more closely the lower-branch cycle of metastable strong streaks. That lower orbit was originally identified from a minimal Couette simulation similar to this one ${ }^{10}$ but, as in the Poiseuille case, the effect is absent from the higher Reynolds numbers.

The Couette boxes with $h^{+}=114$ and $h^{+}=140$ are very similar to each other, although their right-hand tails move steadily away from the lower cycle as the Reynolds number increases, and they are comparable to the full Poiseuille channel at $h^{+} \approx 1000$. Couette flows at these Reynolds numbers are already essentially asymptotic, in agreement with our previous discussion of the experimental evidence.

\section{BURSTING}

While the results just discussed suggest that full-fledged turbulence contains quiescent structures which are essentially identical to the simple solutions of Sec. III, it is clear from the p.d.f.s that it also contains stronger ones, specially as the Reynolds number increases. It is impractical to continue the search for simple solutions to much higher Reynolds numbers than the ones in Sec. III, but it is unlikely that such solutions, if they exist, would turn out to be much stronger than those at the Reynolds numbers presented here. Even simple solutions should eventually scale approximately in wall units. This, and the already-mentioned fact that the turbulent p.d.f.s stretch upwards as the Reynolds number increases, but keep a constant lower bound, raises naturally the question of whether the Reynolds number effects seen in Fig. 11 might be due to unsteady effects.

We already mentioned in the introduction that the question of whether the generation of turbulence in the near-wall layer is best described by permanent coherent structures or by temporally intermittent processes has been discussed often. Near-wall turbulence is clearly not steady, but the question is whether it is closer to a system moving in phase space within the immediate neighborhood of an unstable coherent state, ${ }^{41}$ or to a sequence of intermittent nonlinear excursions 

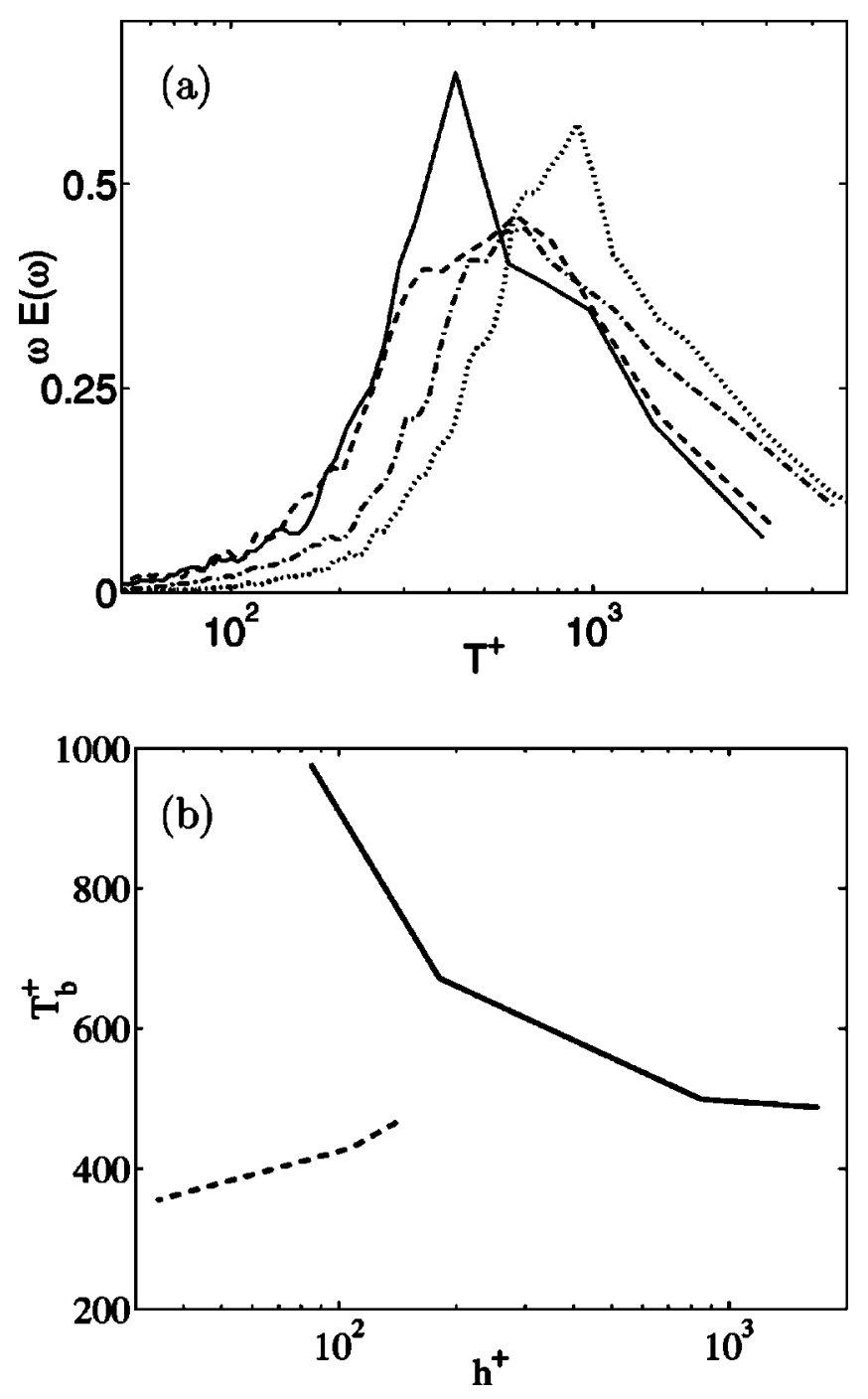

FIG. 12. (a) Premultiplied frequency spectra of the time evolution of the plane-averaged wall shear plotted against the period, for different minimal Poiseuille flows from Table IV. ........, P1; ---, P3; ----, P5; -, P6. (b) Weighted average periods for all the minimal flows in Table IV. - , Poiseuille; ----, Couette.

far from a saddle point along homoclinic or heteroclinic orbits. $^{3,28,42}$

The modern tendency has been to emphasize the first of those views, but evidence for time-dependent bursting has been increasingly difficult to ignore. It is well known that streaks are unstable to sinuous perturbations, ${ }^{7,43,44}$ and lateral oscillations have been implicated in the breakdown of the streaks in simulations of autonomous, ${ }^{15}$ minimal Poiseuille, ${ }^{14}$ and minimal Couette ${ }^{25}$ flows. Schoppa and Hussain ${ }^{44}$ have been specially insistent in urging the reconsideration of the case for time-dependent bursting.

There is no question that minimal flows burst intermittently with fairly well-defined periods. ${ }^{14}$ Frequency spectra of the time histories of their integrated wall shear $\left\langle\omega_{z}\right\rangle(y$ $=0$ ) are shown in Fig. 12(a) and, since there is only one structure in the computational box of minimal simulations, those spectra reflect a temporal evolution.

The mean value of the burst period $T=2 \pi / \omega$ can be defined as the weighted logarithmic average of the periods in the temporal frequency spectrum,

$$
\ln \left(T_{b}\right)=\int \ln (T) E(\omega) \mathrm{d} \omega .
$$

For the Poiseuille flows in Fig. 12(a), the length of the "burst" decreases from $T_{b}^{+} \approx 1000$ at low Reynolds numbers to $T_{b}^{+} \approx 400$ at high $h^{+}$. The longer period is close to the one observed visually in Refs. 14 and 5, and the shorter one is close to the "torus" period in Ref. 5. This shorter value is probably asymptotic for high-Reynolds-number flows, because it changes little between the two highest Reynolds numbers in the figure. Preliminary results for an even higherReynolds-number minimal Poiseuille simulation at $h^{+}$ $\approx 3500$ give the same period (private communication from J. C. del Álamo).

The evolution of the mean burst period for Poiseuille and Couette flows is presented in Fig. 12(b). In the Couette case it increases from $T_{b}^{+} \approx 300$ at the lower Reynolds number, to the same value as in Poiseuille flows at the higher ones, $T_{b}^{+} \approx 400$. The shorter period is not too far from that of the "vortex" orbit in Ref. 10. The convergence of the Poiseuille and Couette flow at high Reynolds numbers recalls the similar one discussed above for the intensity of the fluctuations, and the fact the two flows approach their asymptotes from opposite directions may be related to the similar way in which the production behaves in Fig. 10.

As the minimal flows evolve, their properties generate probability clouds similar to those studied in the preceding section. A useful representation, closely related to the one used in Ref. 10, is to describe the state of the flow by its instantaneous integrated production and dissipation rates over some chosen integration height $\chi$,

$$
P=-\int_{0}^{\chi}\langle\tilde{u} \widetilde{v}\rangle \partial_{y} U \mathrm{~d} y,
$$

and

$$
D=\nu \int_{0}^{\chi}\left\langle|\boldsymbol{\nabla} \widetilde{\mathbf{u}}|^{2}\right\rangle \mathrm{d} y .
$$

The balance of those two quantities determines how energy accumulates or drains from the perturbations.

The joint p.d.f. of those two quantities is shown in Fig. 13(a) for a minimal Poiseuille flow. The arrows in the figure represent the evolution velocity of the system in parameter space, $(\mathrm{d} P / \mathrm{d} t, \mathrm{~d} D / \mathrm{d} t)$. The p.d.f. is compiled as a histogram over $25 \times 25$ bins, and the arrows represent the mean evolution velocity of all the states within a particular bin. Note that the velocities computed in this way are in general lower than the true evolution velocities of the systems within the bin, because of the effect of the vector averaging, but the mean values in the bins near the periphery of the distributions in Fig. 13 are of the same order as their measured standard deviations, and they are therefore probably representative of the true values.

To study the behavior of the flow as it evolves, we divide the $(P-D)$ space into quadrants defined by the principal axes of the probability cloud, as in Fig. 13(a). Note that these are 

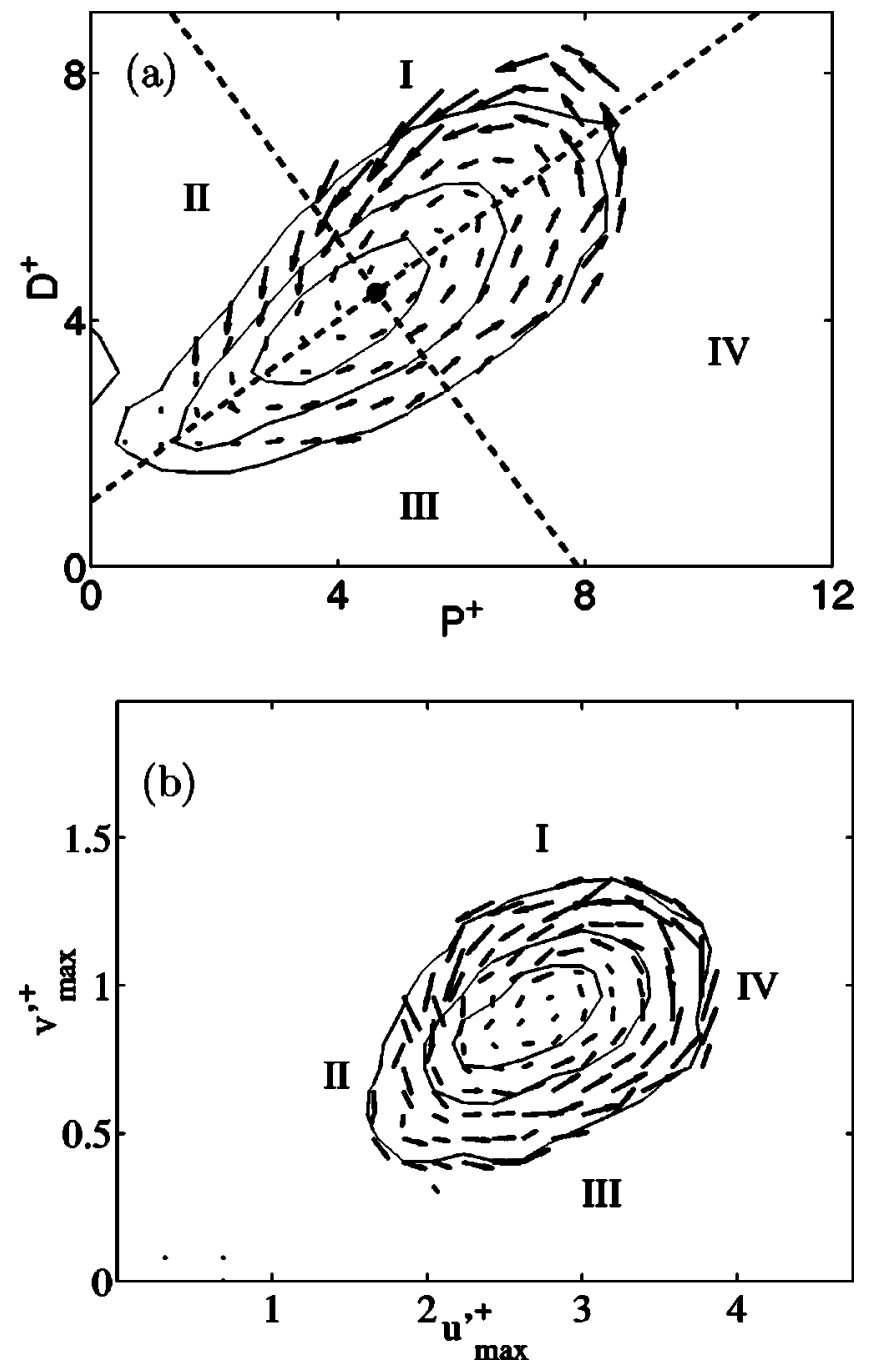

FIG. 13. (a) Evolution velocity and definition of the production-dissipation quadrants. (b) Streak-vortex parameter plane. Minimal flow P3, below $y^{+}$ $=50$.

not the quadrants of the more usual $\left(u^{\prime}-v^{\prime}\right)$ analysis. ${ }^{31,32}$ The present definition essentially guarantees that all the quadrants contain roughly the same number of points, and the subboxes over which our statistics are computed are chosen to be large enough that each of them contains one full sweep and one full ejection. The mean wall-normal velocity over each sub-box is always very close to zero. It is clear from the figure that the flow visits the four quadrants consecutively in the mean, accumulating energy as it moves from III to IV, where $P>D$, and releasing it from I to II.

The residence times of the system in each quadrant can be estimated from its evolution velocity, at least at the periphery of the cloud. The result is that the system crosses quadrant $\mathrm{I}$ in about $T_{\mathrm{I}}^{+} \approx 80$, and the other three quadrants in $T_{\mathrm{II}}^{+} \approx 140, T_{\mathrm{III}}^{+} \approx 150$, and $T_{\mathrm{IV}}^{+} \approx 70$. The total from these estimates agrees well with the periods in Fig. 12, and the implication of the partial crossing times is that the system spends most $(2 / 3)$ of its time at the lower left of the distribution, which is where the simple solutions sit [see Fig. 14(a)], with somewhat shorter excursions into the tail at the right-hand side of the distribution.
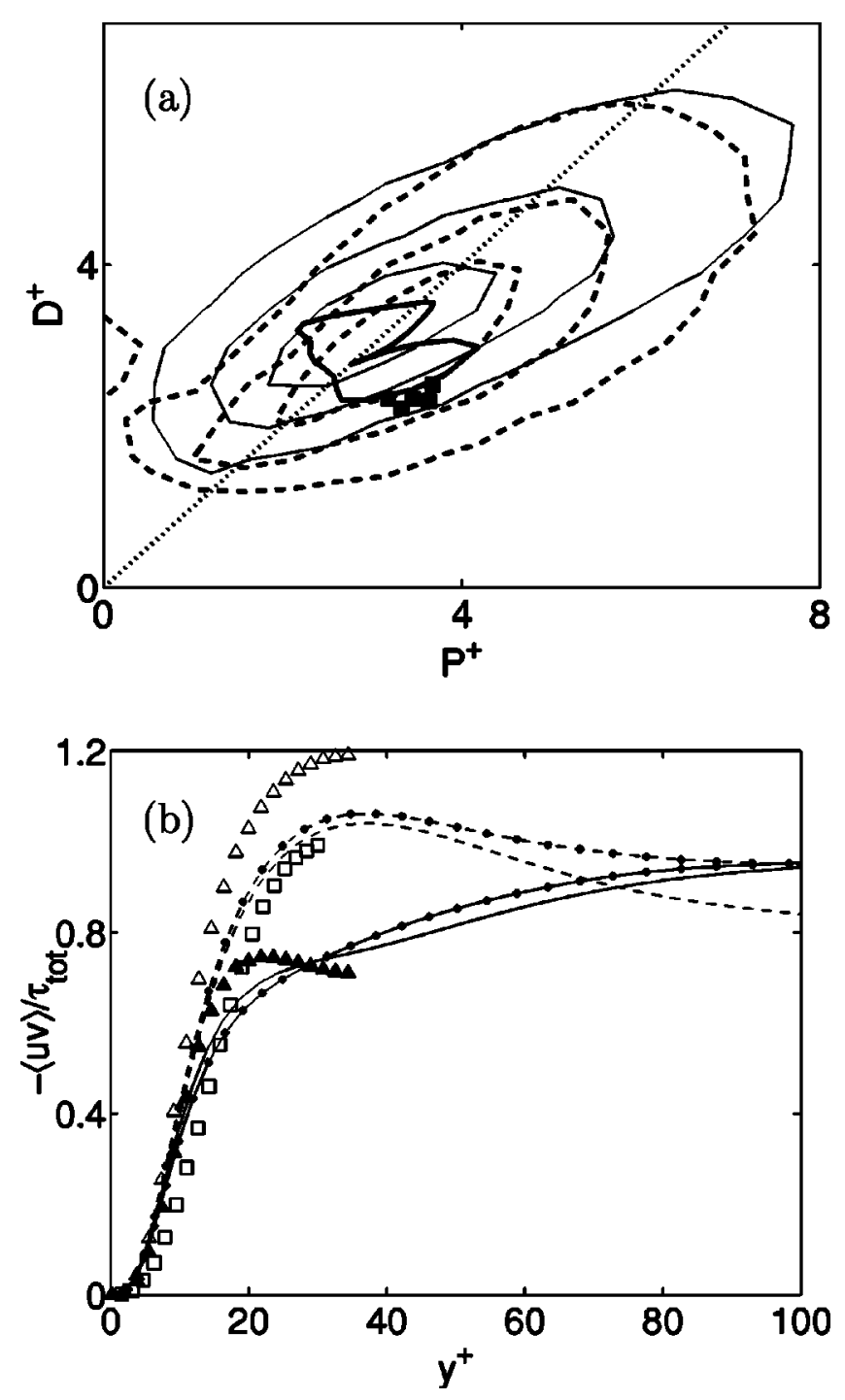

FIG. 14. (a) Joint probability distribution of the production and dissipation. ----, minimal Poiseuille flow P3; - , full Poiseuille flow, both with $h^{+}$ $\approx 180$; the heavy solid line is the Couette cycle O1 in Table III; $\square$, autonomous permanent waves in Table I. The production and dissipation of all these flows are computed below $\chi^{+} \approx 35$. The dotted diagonal is the energy equilibrium $P=D$. (b) Reynolds-stress profiles conditioned for each $(P-D)$ phase, normalized with the total shear stress. - "Dissipation" phase. Quadrants I and II; ----, "production" phase. Quadrants III and IV. Simple lines are minimal Poiseuille flow P3, and lines with heavy dots are a large-box Poiseuille flow, both at $h^{+}=180 ; \square$, average of the five autonomous solutions in Table I; $\triangle$, average of the cycle $\mathrm{O} 1$ when $P>D ; \boldsymbol{\Delta}, \mathrm{O} 1$ for $P$ $<D$.

A similar evolution analysis can be done for other parameter pairs, such as the $\left(u_{\max }^{\prime}-v_{\max }^{\prime}\right)$ plane used in the preceding section and shown in Fig. 13(b). Its sense of rotation is also counterclockwise. There is no one-to-one correspondence between areas near the centers of this and of the $(P-D)$ distribution, but the evolution of their peripheries can be correlated well. Starting from top center in Fig. 13(b), the system moves consecutively through the $(P-D)$ quadrants I-IV, which are now located approximately as indicated in the figure. Note that quadrants II and IV roughly correspond to the left and right wings of the p.d.f.s in Fig. 11.

The general picture that can be derived from plots of this type is consistent with the visual studies of bursting in mini- 
mal channels by previous investigators. ${ }^{14,25,44}$ The flow stays for relatively long times in the dense region of the p.d.f., where it looks like a simple equilibrium state [see Fig. 14(a)]. We saw in Sec. III A that this equilibrium is a balance between the creation of the streaks by the wall-normal velocity of the vortices and their damping by the spanwise velocity. Occasionally the equilibrium is broken and the streaks enter various parts of the bursting cycle. For example, if the vortices grow weaker and the system moves towards the lower edge of Fig. 13(b), the streaks straighten and begin to strengthen, as in the lower-branch solutions, and the system drifts from quadrants III to IV. It follows from the previously cited visual studies that this corresponds to relatively straight streaks with weak vortices which do not grow during this phase of the evolution, as shown by the horizontal motion of the system in Fig. 13(b). This may be the algebraic-growth phase identified in Ref. 44, and it eventually results in streaks that are strong enough to be unstable to the previously mentioned exponential sinuous instability. This limit was identified in Ref. 44 as $u_{\max }^{\prime+} \approx 3-4$ in the present notation, and is consistent with the position of the right-hand edge of the distributions in Fig. 11. This new instability results in vortex growth (IV) and in the eventual destruction of the streak through lateral deformation (I). This in the dissipation-dominated part of the cycle, which results in the final decay of the vortices (II) once their feeding streaks have been destroyed. Note that the vortex decay times found in Ref. 15 by artificially shutting down their vortex production term are of the same order $T^{+} \approx 200$ as those found above for quadrants I and II. Similar initiation scenarios can be postulated starting from other locations along the cycle.

While this correspondence with previous studies of minimal flows is reassuring, the emphasis in this paper should be on the similarities between the minimal channels, the simple structures, and the full turbulent flows. As in the preceding section, the statistics used for the minimal boxes can be defined over minimal sub-boxes of full-scale simulations. The production and dissipation distributions of a mini$\mathrm{mal}$ and of a full simulation at similar Reynolds numbers are compared in Fig. 14(a) below $\chi^{+}=35$, which is a depth of the order of those of the simple solutions. They agree well, as was the case for the $\left(u_{\max }^{\prime}-v_{\max }^{\prime}\right)$ representation in the preceding section, and similar results are found for all the variable pairs that have been tried. Although temporal evolution information is not available for the full simulations, that agreement strongly suggests that the full channel is also bursting. Figure 14(a) also includes data from the autonomous solutions in Table I and from the vortex-dominated orbit $\mathrm{O} 1$ in Table III. They are in the "quiescent" core of the distribution, and it is interesting that the periodic orbit is also traversed counterclockwise by the flow. In fact, the periodic orbit can be considered as a "miniature" bursting cycle in the limit of very low Reynolds numbers, and shares many of the characteristics of higher-Reynolds-number cases. For example, the orbit can be divided into $(P-D)$ quadrants, and $60 \%$ of its time is also spent within the two left-hand quiescent ones.

Note that most of the flows in the figure are not in energy equilibrium over the near-wall layer being considered, which is known to generate a net excess of turbulent energy in real flows. ${ }^{15}$ In particular, note that the autonomous solutions lie near the production branch of the periodic orbit.

The comparison among the different systems can be done in more detail using flow profiles conditioned on the different phases of the bursting cycle. Many of those comparisons are equivalent to the correspondence of the joint p.d.f.s discussed up to now, but one which is difficult to do in that way is Fig. 14(b), which displays the distribution of Reynolds stress in the production and in the dissipation phases of the cycle. The figure also contains data from the vortex-dominated periodic Couette orbit and from the autonomous permanent waves. To facilitate their comparison the Reynolds stress has been normalized with the total stress, i.e., with $u_{\tau}^{2}$ in the autonomous and Couette cases, and with $u_{\tau}^{2}(1-y / h)$ in the Poiseuille flows. The minimal and the full Poiseuille flows also agree well in this representation, and both show a clear distinction between the production and the dissipation phases. Given the differences in Reynolds numbers, the agreement of the other solutions is also good. In particular, the autonomous permanent waves are again near the production branch. One surprise is that the Reynolds stress near the wall is predominantly generated during the quiescent and streak-growth production phase, while the vortex-dominated states in quadrants I and II carry much less stress in that region. This is clearly because the temporal burst destroys the coherence of the near-wall structures, but it goes against the often-quoted rule that the Reynolds stress is carried by the bursts.

Other plots such as this one also suggest that the activity during the dissipation phase of the cycle moves away from the wall, which brings to mind the large-scale structures identified by different investigators in the logarithmic layer. ${ }^{34,45-48}$ It has been suggested that those structures could be self-propelled coherent packets of hairpins vortices, ${ }^{49}$ and it is tempting to speculate that the bursts identified here could be the initial triggers for such packets.

There are not at the moment enough data on the distance between vortex packets to test this assumption, and the packets themselves, being predominantly above the buffer layer, are outside the scope of the present paper, but it is interesting to note that the temporal information obtained from the minimal simulations can be related to the length of the bufferlayer structures of the full flows. If we assume that the advection velocity of features in this part of the flow ${ }^{50}$ is $c^{+}$ $\approx 10$, a bursting period of $T_{p}^{+}=400$ corresponds to a length of $\lambda_{x}^{+} \approx 4000$, which is in good agreement with the observed length of "composite" streaks in the buffer layer. ${ }^{17}$ Such streaks can be expected to contain several vortex systems, on average one every $\lambda_{x}^{+} \approx 400$, but not all of them are "excited." If we, for example, define as excited systems those in quadrants I and IV in Fig. 13(a), the fraction of excited boxes would be proportional to $\left(T_{\mathrm{I}}+T_{\mathrm{IV}}\right) / T_{p} \approx 0.33$, The implied distance between consecutive excited vortex systems along a streak is $\lambda_{x}^{+} \approx 1200$, which is also in good agreement with the measured length of the coherent part of each streak. ${ }^{17}$ Note that these lengths imply that, if the bursts are really the triggers for the logarithmic-layer vortex packets, the mean distance between such packets would turn out to be of the order 
of $\lambda_{x}^{+} \times \lambda_{z}^{+} \approx 1200 \times 300$, and some interaction among neighboring packets should be expected once they grow to approximately such size.

\section{CONCLUSIONS}

We have shown that several known "simple" solutions to the Navier-Stokes equations, particularly those which correspond to permanent waves and to limit cycles in autonomous and Couette flows, can be classified into upper- and lowerbranch families which agree fairly well with the corresponding branches of the Couette waves found by Nagata. ${ }^{1}$ The velocity statistics within each branch are reasonably similar to each other, even though the base flows are quite different. The upper branch consists of weak streaks with strong vortices, and the lower one has much stronger streaks and weaker vortices.

Turbulence in minimal Poiseuille or Couette flows stays close to the vortex-dominated upper solutions, and fully turbulent simulations in large boxes, when analyzed over subboxes of minimal size, have statistics which are also consistent with those solutions. In particular, the range of spanwise wavelengths for which the steady solutions exist is in the near $\lambda_{z}^{+} \approx 100$ for all the Reynolds numbers investigated, and the streamwise wavelengths for upper-branch solutions is bounded below by $\lambda_{x}^{+} \approx 250$, both in agreement with comparable length scales of fully developed wall turbulence. We have argued that the structures of real turbulence which are represented by these solutions are the active nonlinear scales of the viscous and buffer region, which constitute the autonomous self-regenerating "core" of this part of the flow.

Kawahara and Kida ${ }^{10}$ found that minimal Couette turbulence at very low Reynolds numbers intermittently visits the lower-branch solutions, but the same is not true in flows at somewhat higher Reynolds numbers or in larger boxes. What remains of this behavior is a tendency of minimal simulations to make occasional extreme excursions either towards lower-branch solutions or towards laminar states, but even those are much less common in turbulence in large boxes.

We have interpreted this to mean that the lower branch, which has been linked to bypass transition to full turbulence, ${ }^{3,10,40}$ represents a metastable state which minimal turbulence can reach before retransitioning, but that these events are not allowed at higher Reynolds numbers, or in larger boxes, because of the higher level of ambient perturbations.

There is a general tendency for the statistics of minimal or full flows to extend to intensities which are higher than those of the simple solutions as the Reynolds number increases, but this trend saturates for $h^{+} \gtrsim 1000$ in Poiseuille flow, and much sooner in the Couette case. The limiting state is the same for both types of flows.

We have given evidence that this higher intensities are associated with intermittent temporal bursting both in minimal and in full flows, with periods which tend to $T_{b}^{+} \approx 400$ at high Reynolds numbers. This phenomenon is different from the passing of individual coherent structures, which are well described by the simple solutions mentioned above. The temporal bursting involves the destabilization of those struc- tures, probably by two different processes. The streaks initially grow in intensity without a corresponding strengthening of the vortices, and later undergo a faster instability in which stronger vortices are created, move away from the wall, and eventually destroy the streak. We have presented statistics of both minimal and full flows conditioned on different parts of the cycle. Contrary to the oftenquoted belief, these temporal bursts carry relatively little Reynolds stress near the wall. The stress in the buffer layer is predominantly carried by the "steady" structures. The periodic orbit identified in Ref. 10 has been shown to be a weaker version of the bursting cycle, sharing with it many of its characteristics.

The temporal information obtained from the minimal flows can be used to predict the spatial scales of the streaks in the buffer layer of full flows, which agrees well with observed values. It is speculated that the burst could act as triggers for the large-scale vortex packets that have been described elsewhere in the logarithmic layer, and it is shown that this would lead to specific predictions regarding their spacing and their eventual evolution.

\section{ACKNOWLEDGMENTS}

The authors cordially thank Professor S. Toh and Dr. T. Itano for providing the original data of their traveling-wave and periodic-like solutions, and J. C. del Álamo for the time histories of the high-Reynolds-number minimal channels P5 and P6 in Table IV, and for providing and postprocessing the data for the full simulations. This work was started when G.K. participated in the 2002 Summer Program of the Center for Turbulence Research of Stanford University and NASA Ames. The financial support and kind hospitality of the CTR are gratefully acknowledged. G.K. and M.S. appreciate helpful discussions with Professor S. Yanase in the development of a numerical code for an equilibrium solution. This work was supported in part by a Grant-in-Aid for Scientific Research (B, C) from the Japan Society for the Promotion of Science, by the Center of Excellence for Research and Education on Complex Functional Mechanical Systems (COE program of the Ministry of Education, Culture, Sport, Science and Technology of Japan), and by the Spanish CICYT Grant No. DPI2003-03434. M.P.S. was supported in part by a fellowship from the EU TMR network on Mixing and Stirring.

\footnotetext{
${ }^{1}$ M. Nagata, "Three-dimensional finite-amplitude solutions in plane Couette flow: Bifurcation from infinity," J. Fluid Mech. 217, 519 (1990).

${ }^{2} \mathrm{~F}$. Waleffe, "Homotopy of exact coherent structures in plane shear flows," Phys. Fluids 15, 1517 (2003).

${ }^{3} \mathrm{~S}$. Toh and T. Itano, "On the regeneration mechanism of turbulence in the channel flow," Proceedings of IUTAM Symposium on Geometry and Statistics of Turbulence, edited by T. Kambe, T. Nakano, and T. Miyauchi (Kluwer, Dordrecht, 2001), p. 305.

${ }^{4} \mathrm{~F}$. Waleffe, "Exact coherent structures in channel flow," J. Fluid Mech. 435, 93 (2001).

${ }^{5}$ J. Jiménez and M. P. Simens, "Low-dimensional dynamics in a turbulent wall flow," J. Fluid Mech. 435, 81 (2001).

${ }^{6} \mathrm{~F}$. Waleffe, "Three-dimensional coherent states in plane shear flows," Phys. Rev. Lett. 81, 4140 (1998).

${ }^{7}$ G. Kawahara, J. Jiménez, M. Uhlmann, and A. Pinelli, "Linear instability of a corrugated vortex sheet-a model for streak instability," J. Fluid Mech. 483, 315 (2003).
} 
${ }^{8}$ D. D. Stretch, "Automated pattern eduction from turbulent flow diagnostics," CTR Annual Research Briefs 1990 (NASA Ames/Stanford University, Stanford, 1990), p. 145.

${ }^{9}$ J. Jeong, F. Hussain, W. Schoppa, and J. Kim, "Coherent structures near the wall in a turbulent channel flow," J. Fluid Mech. 332, 185 (1997).

${ }^{10}$ G. Kawahara and S. Kida, "Periodic motion embedded in plane Couette turbulence: Regeneration cycle and burst," J. Fluid Mech. 449, 291 (2001).

${ }^{11}$ H. T. Kim, S. J. Kline, and W. C. Reynolds, "The production of turbulence near a smooth wall in a turbulent boundary layer," J. Fluid Mech. 50, 133 (1971).

${ }^{12}$ G. R. Offen and S. J. Kline, "A proposed model for the bursting process in turbulent boundary layers," J. Fluid Mech. 70, 209 (1975).

${ }^{13}$ S. K. Robinson, "Coherent motions in the turbulent boundary layer," Annu. Rev. Fluid Mech. 23, 601 (1991).

${ }^{14}$ J. Jiménez and P. Moin, "The minimal flow unit in near wall turbulence," J. Fluid Mech. 225, 221 (1991).

${ }^{15}$ J. Jiménez and A. Pinelli, "The autonomous cycle of near wall turbulence," J. Fluid Mech. 389, 335 (1999).

${ }^{16}$ G. Kawahara, J. Jiménez, M. Shiba, and M. Simens, "Characterization of near-wall turbulence in terms of equilibrium and periodic solutions," Proceedings of CTR Summer Program 2002 (NASA Ames/Stanford University, Stanford, 2002), p. 179.

${ }^{17}$ J. Jiménez, J. C. del Álamo, and O. Flores, "The large-scale dynamics of near-wall turbulence," J. Fluid Mech. 505, 179 (2004).

${ }^{18}$ J. Kim, P. Moin, and R. Moser, "Turbulence statistics in fully developed channel flow at low Reynolds number," J. Fluid Mech. 177, 133 (1987).

${ }^{19}$ R. Corral and J. Jiménez, "Fourier/Chebyshev methods for the incompressible Navier Stokes equations in infinite domains," J. Comput. Phys. 121, 261 (1995)

${ }^{20} \mathrm{U}$. Ehrenstein and W. Koch, "Three-dimensional wavelike equilibrium states in plane Poiseuille flow," J. Fluid Mech. 228, 111 (1991).

${ }^{21}$ M. Nagata, "Bifurcations in Couette flow between almost corotating cylinders," J. Fluid Mech. 169, 229 (1986).

${ }^{22}$ M. Nagata, "On wavy instabilities of the Taylor-vortex flow between corotating cylinders," J. Fluid Mech. 188, 585 (1988).

${ }^{23}$ R. D. Moser, J. Kim, and N. N. Mansour, "Direct numerical simulation of turbulent channel flow up to $\mathrm{Re}_{\tau}=590$," Phys. Fluids 11, 943 (1999).

${ }^{24}$ C. R. Smith and S. P. Metzler, "The characteristics of low-speed streaks in the near-wall region of a turbulent boundary layer," J. Fluid Mech. 129, 27 (1983).

${ }^{25}$ J. M. Hamilton, J. Kim, and F. Waleffe, "Regeneration mechanisms of near-wall turbulence structures," J. Fluid Mech. 287, 317 (1995).

${ }^{26} \mathrm{~K}$. Coughlin, J. Jiménez, and R. Moser, "Instability of streamwise vortices in plane channel flows," Proceedings of CTR Summer Program 1994 (NASA Ames/Stanford University, Stanford, 1994), p. 229.

${ }^{27}$ R. M. Clever and F. H. Busse, "Tertiary and quaternary solutions for plane Couette flow," J. Fluid Mech. 344, 137 (1997).

${ }^{28} \mathrm{~S}$. Toh and T. Itano, "A periodic-like solution in channel flow," J. Fluid Mech. 481, 67 (2003).
${ }^{29}$ J. Neu, "The dynamics of stretched vortices," J. Fluid Mech. 143, 253 (1984).

${ }^{30}$ P. Orlandi and J. Jiménez, "On the generation of turbulent wall friction," Phys. Fluids 6, 634 (1994).

${ }^{31}$ J. M. Wallace, H. Eckelmann, and R. S. Brodkey, "The wall region in turbulent shear flow," J. Fluid Mech. 54, 39 (1972).

${ }^{32}$ W. W. Willmarth and S. S. Lu, "Structure of the Reynolds stress near the wall," J. Fluid Mech. 55, 65 (1972).

${ }^{33}$ J. C. del Álamo and J. Jiménez, "Spectra of very large anisotropic scales in turbulent channels," Phys. Fluids 15, L41 (2003).

${ }^{34}$ J. C. del Álamo, J. Jiménez, P. Zandonade, and R. D. Moser, "Scaling of the energy spectra of turbulent channels," J. Fluid Mech. 500, 135 (2004).

${ }^{35}$ D. B. DeGraaf and J. K. Eaton, "Reynolds number scaling of the flat-plate turbulent boundary layer," J. Fluid Mech. 422, 319 (2000).

${ }^{36}$ K. H. Bech, N. Tillmark, P. H. Alfredsson, and H. I. Andersson, "An investigation of turbulent plane Couette flow at low Reynolds numbers," J. Fluid Mech. 286, 291 (1995).

${ }^{37}$ T. Wei and W. W. Willmarth, "Reynolds-number effects on the structure of a turbulent channel flow," J. Fluid Mech. 204, 57 (1989).

${ }^{38}$ M. M. M. El Telbany and A. J. Reynolds, "The structure of turbulent plane Couette flow,” J. Fluids Eng. 104, 367 (1982).

${ }^{39}$ J. Komminaho, A. Lundbladh, and A. V. Johansson, "Very large structures in plane turbulent Couette flow," J. Fluid Mech. 320, 259 (1996).

${ }^{40} \mathrm{~T}$. Itano and $\mathrm{S}$. Toh, "The dynamics of bursting process in wall turbulence," J. Phys. Soc. Jpn. 70, 703 (2001).

${ }^{41}$ F. Waleffe, "Transition in shear flows. Nonlinear normality versus nonnormal linearity," Phys. Fluids 7, 3060 (1995).

${ }^{42}$ N. Aubry, P. Holmes, J. L. Lumley, and E. Stone, "The dynamics of coherent structures in the wall region of a turbulent boundary layer," $\mathrm{J}$. Fluid Mech. 192, 115 (1988).

${ }^{43}$ F. Waleffe, "On a self-sustaining process in shear flows," Phys. Fluids 9, 883 (1997).

${ }^{44}$ W. Schoppa and F. Hussain, "Coherent structure generation in near-wall turbulence," J. Fluid Mech. 453, 57 (2002).

${ }^{45}$ A. E. Perry, S. Henbest, and M. S. Chong, "A theoretical and experimental study of wall turbulence," J. Fluid Mech. 165, 163 (1986).

${ }^{46} \mathrm{M}$. Hites, "Scaling of high-Reynolds number turbulent boundary layers in the National Diagnostic Facility," Ph.D. thesis, Illinois Institute of Technology, 1997.

${ }^{47}$ J. Jiménez, "The largest structures in turbulent wall flows," CTR Annual Research Briefs 1998 (NASA Ames/Stanford University, Stanford, 1998), p. 137.

${ }^{48}$ K. Kim and R. Adrian, "Very large-scale motion in the outer layer," Phys. Fluids 11, 417 (1999).

${ }^{49}$ R. J. Adrian, C. D. Meinhart, and C. D. Tomkins, "Vortex organization in the outer region of the turbulent boundary layer," J. Fluid Mech. 422, 1 (2000).

${ }^{50} \mathrm{~J}$. Kim and F. Hussain, "Propagation velocity of perturbations in channel flow," Phys. Fluids A 5, 695 (1993). 